\title{
Experimental Validation of Fly-Wheel Passive Launch and On-Orbit Vibration Isolation System by Using a Superelastic SMA Mesh Washer Isolator
}

\author{
Seong-Cheol Kwon, ${ }^{1}$ Mun-Shin Jo, ${ }^{2}$ and Hyun-Ung $\mathrm{Oh}^{1}$ \\ ${ }^{1}$ Space Technology Synthesis Laboratory, Department of Aerospace Engineering, Chosun University, 375 Seosuk-dong, \\ Dong-gu, Gwangju 501-759, Republic of Korea \\ ${ }^{2}$ Mechanical Design Team, Hanwha Systems, 304 Cheoin-gu, Yongin 449-886, Republic of Korea \\ Correspondence should be addressed to Hyun-Ung Oh; ohu129@chosun.ac.kr
}

Received 29 January 2017; Accepted 5 July 2017; Published 17 August 2017

Academic Editor: Paolo Tortora

Copyright (c) 2017 Seong-Cheol Kwon et al. This is an open access article distributed under the Creative Commons Attribution License, which permits unrestricted use, distribution, and reproduction in any medium, provided the original work is properly cited.

\begin{abstract}
On-board appendages with mechanical moving parts for satellites produce undesirable micro-jitters during their on-orbit operation. These micro-jitters may seriously affect the image quality from high-resolution observation satellites. A new application form of a passive vibration isolation system was proposed and investigated using a pseudoelastic SMA mesh washer. This system guarantees vibration isolation performance in a launch environment while effectively isolating the micro-disturbances from the on-orbit operation of jitter source. The main feature of the isolator proposed in this study is the use of a ring-type mesh washer as the main axis to support the micro-jitter source. This feature contrasts with conventional applications of the mesh washers where vibration damping is effective only in the thickness direction of the mesh washer. In this study, the basic characteristics of the SMA mesh washer isolator in each axis were measured in static tests. The effectiveness of the design for the new application form of the SMA mesh washer proposed in this study was demonstrated through both launch environment vibration test at qualification level and micro-jitter measurement test which corresponds to on-orbit condition.
\end{abstract}

\section{Introduction}

The quality of high-resolution images obtained from earth observation satellites can be degraded by undesirable microdisturbances induced by on-board appendages that have mechanical moving parts, such as reaction wheel assembly (RWA) [1], control moment gyro [2], cryogenic cooler [3], and gimbal-type antenna [4]. Among the various vibration disturbance sources, micro-jitter caused by the RWA is known to have the greatest effect on the performance of the high-resolution optical payloads. The major causes of the disturbances from the RWA are rotor imbalance, bearing imperfections, motor disturbances, and motor driven errors [5]. Recently, micro-jitter level is becoming quietly lower by high precise balancing of the spinning rotor of the RWAs. However, these micro-jitters may still seriously affect the image quality of high-resolution observation satellites as the acceptable amplitudes of the micro-jitters are also becoming much lower to meet strict mission requirements.

To ensure the performance of observation satellites, it is important task to isolate the micro-jitters from the RWAs. This isolation can be accomplished by mounting the RWAs on a vibration isolator with low stiffness by attenuating the transmitted vibration to the main payload structures, which are sensitive to the micro-jitters. Kamesh et al. [6] proposed a low-frequency flexible platform that consisted of folded continuous beams for passive RWA vibration isolation. The effectiveness of the design was confirmed through tests conducted by mounting the RWA on several configurations of the flexible platform. The results indicated that this passive isolation platform is capable of mitigating the disturbance vibration by serving as an isolation mount, not only for RWAs but also for other disturbance sources in spacecraft. 
Davis et al. [7] proposed a passive viscous-damped strut (D-strut) with a very low fundamental frequency of $1.5 \mathrm{~Hz}$ to isolate disturbance forces and torques from a Honeywell HM-1800 RWA with a frequency band from 2 to $300 \mathrm{~Hz}$. In addition, it has been widely used in various space programs including the Hubble mission. Zhang et al. [8] proposed a new vibration isolation system that contains a multistrut vibration isolation platform with multiple tuned mass dampers to achieve RWA micro-jitter isolation. The numerical simulation results demonstrated that the RWA disturbances were effectively attenuated by the newly proposed vibration isolation system while simultaneously validating its safety performance when the speed of the RWA crossed the resonance frequency.

Oh et al. [9] proposed a RWA variable-damping isolator using a biometal fiber valve with the characteristics of low power consumption, which can select the suitable damping according to the rotating speed of the RWA in order to minimize the transmitted force to the main payload structure. Additionally, the effectiveness of the design was demonstrated by the micro-jitter measurement test of a dummy RWA supported by the isolator on an air-floating type microdisturbance measurement device [10].

Several types of passive RWA vibration isolation systems [11-14], including the aforementioned applications, provide excellent micro-jitter isolation performance. However, the structural safety of the RWA cannot be guaranteed under the severe vibration conditions experienced in a launch environment when supported by a low-stiffness isolator. If the isolator supporting the RWA is rigidly fixed by a holding and release mechanism during launch and then released in an orbit, the structural safety problems can be readily solved. However, this approach increases the system complexity, reduces its reliability, and increases the mass of the total system. In addition, if there is a problem in activating the release mechanism, then the micro-vibrations cannot be expected anymore. Therefore, Oh et al. [15] developed a passive launch and on-orbit vibration isolation system (PLOVIS) for the purpose of vibration attenuation of a cryogenic cooler under both launch and on-orbit vibration environments. The effectiveness of the isolator was demonstrated by launch environment tests and a micro-vibration measurement test of the cooler. Oh et al. $[16,17]$ also proposed a strategy to use a compressed shape memory alloy (SMA) mesh washer on the existing cooler isolation system [15] to attenuate the microvibration induced by cryocooler operation in on-orbit environment. Additionally, the SMA washer effectively enhanced the vibration attenuation performance during severe launch environments when compared to the conventional isolation system [15].

In this study, we investigate the possibility of achieving a novel launch and on-orbit vibration isolation system which guarantees the vibration isolation performance in a launch environment without the requirement of a holding and release mechanism, while effectively isolating the micro-jitter disturbances from the RWAs on-orbit. For this, we focused on a compressed mesh washer that exploits the pseudoelasticity of SMA wire, as proposed by Youn et al. [18]. The largely deformed SMA mesh washer can recover its original shape without plastic deformation upon unloading because of its pseudoelastic or superelastic SMA behavior, which is caused by stress-induced phase transformation from austenite to martensite. The large damping of the mesh washer is also one of the great advantages of the SMA mesh washer. The strategy to achieve the design goal of this study is to use the radial direction of the ring-type SMA mesh washer as a main axis to support the vibration sources. This strategy is in contrast with the applications of conventional mesh washers [16-20], in which vibration damping is effective only in the thickness direction of the washer.

In the previous study, the effectiveness of the new application form of the SMA mesh washer using a radial direction of the ring-type mesh washer was investigated [21, 22]. Kwon et al. proposed a novel pseudoelastic gear that utilized the ring-type SMA mesh washer as a spring-blade to bridge the output shaft of the motor with an existing gear wheel [21]. This application was researched and developed for attenuating the micro-jitter induced by stepper motor activation of the twoaxis gimbal antenna. This application makes it possible to achieve a low rotational stiffness and high damping characteristics, which are the main contributors to the micro-jitter isolation capability. In addition, the pseudoelasticity of the SMA mesh washer guaranteed the structural safety of the gear itself even under unexpected circumstances such as an overdriving torque condition. Its effectiveness was verified through the micro-jitter measurement tests using a gimbal-type antenna.

Oh et al. proposed a whole antenna isolation system employing a ring-type SMA mesh washer that supports the gimbal-type antenna [22]. This application makes it possible to achieve a relatively much lower rotational stiffness of the mesh washer than in the axial direction. The rotational axis of the azimuth gear wheel of the antenna corresponds to the main jitter sources induced by stepper motor activation and an imperfect intermeshed harmonic-drive-gear configuration. Therefore, the weak rotational stiffness of the mesh washer isolator enhances the performance of the micro-jitter isolation. However, the effectiveness of the vibration isolation performance under a launch environment, where the vibration level is quietly much higher than the on-orbit microvibration condition, was not verified in the previous study.

In this study, we verified the feasibility of the new application form of a superelastic SMA mesh washer by applying it to a RWA vibration isolation system. The objective of the design is to guarantee the structural safety of both the jitter sources and the isolator itself in severe launch environments without requiring an additional holding and release mechanism and while effectively isolating the RWA-induced micro-jitter. To measure the basic characteristics of the ring-type SMA mesh washer isolator, which is different application form with the conventional washers, we performed a static test at various compressive and elongation ranges of the isolator, in both the axial and lateral directions. Based on the static test results, we measured the basic characteristics of the isolator in each axis. To confirm the micro-jitter isolation capability by using the new form of the compressed SMA mesh washer isolator, a micro-jitter measurement test of the RWA was performed on a Kistler table with and without the isolation system. In this paper, launch vibration environment tests, such as sine, 


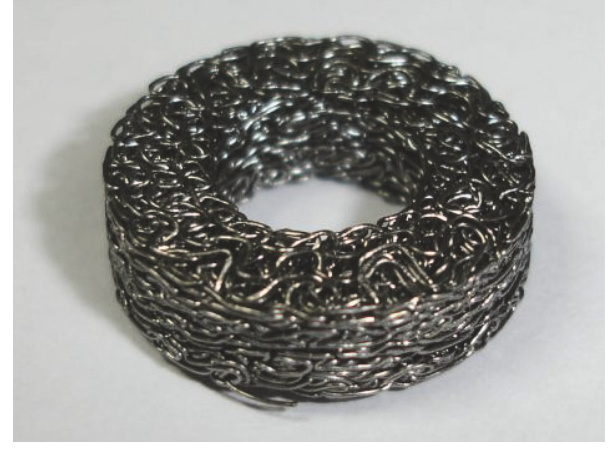

FIGURE 1: Pseudoelastic compressed SMA mesh washer isolator [21].

random vibrations, and shock tests, were also performed at the qualification level. These test results indicated that the launch and on-orbit vibration can be significantly attenuated by using the newly proposed application of the compressed SMA mesh washer. The isolation system guarantees both the structural safety of the RWA during the launch environment and the micro-jitter isolation of RWA during on-orbit conditions.

\section{Passive Launch and On-Orbit Vibration Isolator Using a SMA Mesh Washer}

2.1. Basic Characteristics of Superelastic SMA Mesh Washer. To achieve a passive launch and on-orbit vibration isolator that does not require a hold and release mechanism, we focused on the superelastic behavior of the compressed SMA mesh washer as shown in Figure 1 [21]. The SMA mesh washer was manufactured by pressing a knitted wire mesh by using a mold of the required shape. The SMA mesh washer used in this study was fabricated from SE508 superelastic SMA wire with diameter of $0.2 \mathrm{~mm}$. The outer diameter, inner diameter, and thickness of the SMA mesh washer were $16 \mathrm{~mm}, 8 \mathrm{~mm}$, and $5 \mathrm{~mm}$, respectively. The density and mass of the mesh washer were $1.7 \mathrm{~g} / \mathrm{cm}^{3}$ and $1.88 \mathrm{~g}$. The density was $50 \%$ lower than that of the mesh washer used by Youn et al. [18] and Oh et al. $[16,17,22]$.

Figure 2 shows an example of the representative static test results of the SMA mesh washer shown in Figure 1 during compressive loading and unloading in the thickness direction of the washer [21]. The test results indicate that a largely deformed SMA mesh washer in the thickness direction with a displacement of $2.5 \mathrm{~mm}$ can recover its original shape without plastic deformation upon unloading due to its pseudoelastic or superelastic SMA characteristics. This behavior is a material effect in which the original shape restores itself upon unloading after any large deformation over $10 \%$, yet without being plastically deformed. This is one of the great features of the superelastic SAM mesh washer, which are rarely observed in ordinary metal materials. In addition, the SMA mesh washer also provided better damping capacity than any conventional metal washer isolator [18]. Youn et al. [18] proposed to use the SMA mesh washer as a pyroshock isolator instead of using a general metal washer. However,

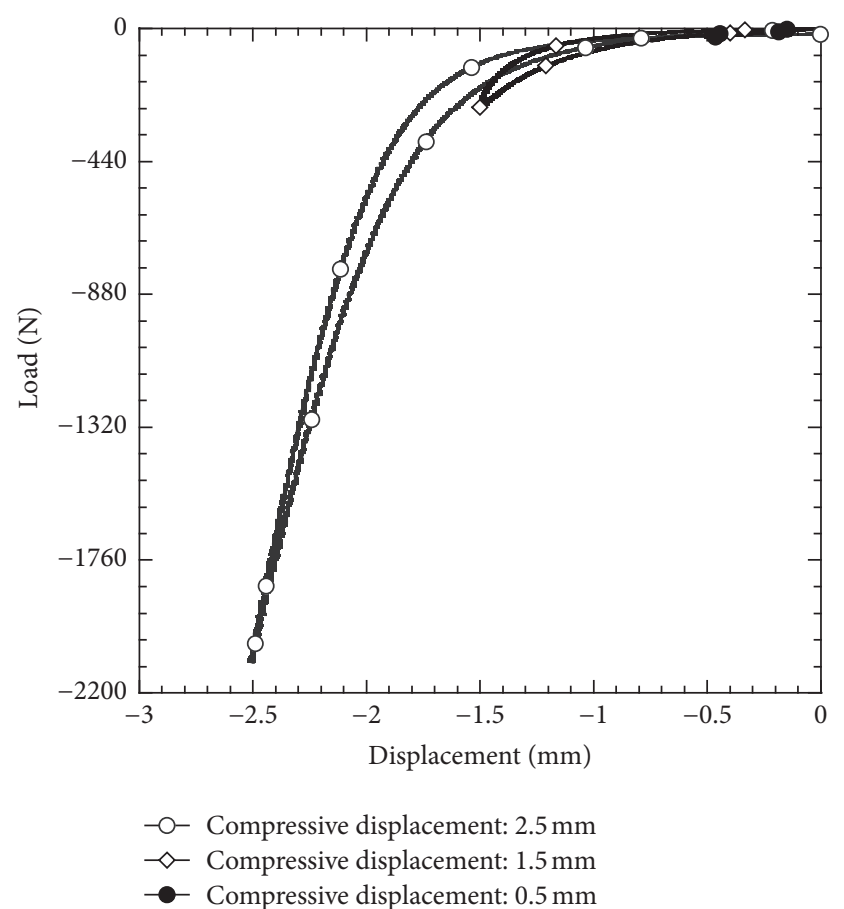

FIGURE 2: Representative load-displacement relationships for a SMA mesh washer along thickness direction [21].

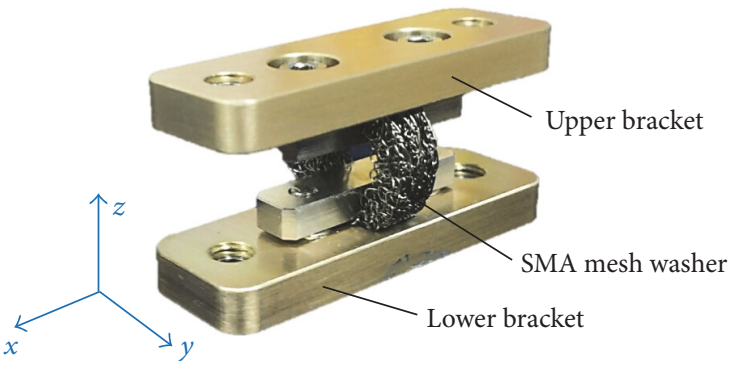

FIGURE 3: New application form of the ring-type SMA mesh washer isolator.

this application is effective only in the thickness direction of the washer. In addition, the characteristics of the washer may be changed by implementing a fastening torque in a real assembly process to satisfy the torque requirement. It is difficult to apply the conventional mesh washer for RWA micro-jitter isolation to achieve objectives of this study.

To overcome the drawbacks discussed above, we proposed a new application form of the SMA mesh washer, as shown in Figure 3. Unlike the applications of a conventional mesh washer, in which vibration damping is effective only in the thickness direction of the washer, this application uses a radial direction of the ring-type mesh washer as a main axis to support the RWA. This application achieved a relatively much lower rotational stiffness for the mesh washer than using the axial direction. This lower rotational stiffness may contribute to micro-jitter isolation as the rotational axis corresponds to the main micro-jitter sources of the RWA. 


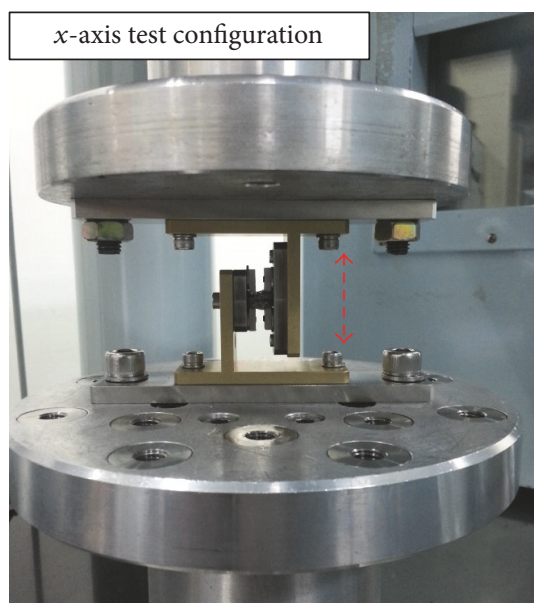

FIGURE 4: Representative static loading test configuration of the ring-type SMA mesh washer along with $x$-axis.

Static loading tests were performed to confirm the basic characteristics of the ring-type SMA mesh washer isolator on each axis. Then, the output data obtained from the tests was used to determine the accommodation of the ring-type SMA mesh washer isolator on the RWA. Figure 4 shows the representative test set-up for the static loading test of the ring-type SMA mesh washer isolator along the $x$-axis. During the test, three repeated cycles of elongation and contraction loading were applied to the washer with various displacement ranges based on increments of $\pm 1 \mathrm{~mm}$ along the $x$-, $y$-, and $z$ axes, respectively. The test conditions for the temperature and strain rate were $20^{\circ} \mathrm{C}$ and $20 \mathrm{~mm} / \mathrm{min}$, respectively.

Figures 5(a) and 5(b) represent the load-displacement relationships obtained from the static loading test of the SMA mesh washer isolator. In this figure, the results are plotted on the $x$-, $y$-, and $z$-axes for displacement ranges of $\pm 1 \mathrm{~mm}$ and $\pm 3 \mathrm{~mm}$. The test results indicated the mesh washer completely recovered its original shape without any plastic deformation in the maximum displacement range of $\pm 3.0 \mathrm{~mm}$. The hysteresis induced by ring-type SMA mesh washer during the loading-unloading cycle indicates that the SMA mesh washer experienced better energy absorption because of its pseudoelastic shape memory effects and the energy dissipation characteristics of the mesh isolator itself. The area enclosed by hysteretic curves is different for each axis, which means that the isolator has different damping capacities.

The static test results along the $z$-axis of the isolator showed substantial variation in the slopes in accordance with the deflection range of the mesh washer. The slope of the isolator decreases as the deflection of the washer increases. This is especially apparent along the $z$-axis. This phenomenon might be related to the direction of the woven pseudoelastic SMA fibers that comprise the mesh. The SMA mesh washer used in this study was fabricated through a pressing process of the knitted pseudoelastic SMA wire mesh, which is then stacked in layers to produce the mesh washer.

Therefore, the geometry of the mesh washer causes it to be more susceptible to delamination between the stacked layers of the compressed washer during loading in the $z$-axis than during loading in the other axes. This is the main reason for the substantial variation in stiffness with regard to the deflection range of the mesh washer. However, plastic deformation, or any permanent damage due to local delamination, was not observed during the static test along the $z$-axis. Additionally, the washer experiencing repeated large deflections of $\pm 3 \mathrm{~mm}$ during static loading tests completely recovered its original shape without any plastic deformation of the washer due to the pseudoelastic SMA behavior.

From the test results, we calculated the equivalent damping and stiffness values. The equivalent damping can be achieved by calculating the area enclosed by the hysteresis curves and the equivalent stiffness of the isolator. In the calculation for obtaining the equivalent damping, an equivalent linearization method where the nonlinear phenomena in slopes and hysteresis are regarded as linear ones in accordance with the energy-balancing principle is used [23]. To assess the damping capacity of the isolator along each axis, the equivalent linear damping coefficient of $\zeta_{\mathrm{eq}}$ is calculated using the following equation:

$$
\zeta_{\mathrm{eq}}\left(a_{0}\right)=\frac{\Delta E\left(a_{0}\right)}{2 \pi a_{0}^{2} K_{\mathrm{eq}}},
$$

where $\Delta E$ is the area enclosed by the hysteresis curve and $a_{0}$ is the displacement amplitude. The equivalent stiffness values of $K_{\text {eq }}$ for various displacement ranges are calculated by linear fitting method by using the slopes of the load-displacement relationships shown in Figure 5.

Figure 6 shows the estimated equivalent stiffness and damping values of $K_{\mathrm{eq}}$ and $\zeta_{\mathrm{eq}}$ under various displacement ranges along each axis. The $K_{\mathrm{eq}}$ along the $z$-axis indicates the highest values compared to the other axes. For instance, the stiffness value with a $\pm 1 \mathrm{~mm}$ deflection of the isolator in the $z$-axis is 9.4 times larger than the value obtained from the $x$ axis, which shows the lowest stiffness value. However, it can be observed that the stiffness in the $z$-axis relatively largely declines with an increase in deflection range, compared to the other axes, owing to the aforementioned reason for the direction of the woven SMA fibers that are meshed together. The calculated equivalent damping values of $\zeta_{\text {eq }}$ with respect to the deflection ranges of the isolator in each axis are also plotted in Figure 6. The results indicate that the maximum value of $\zeta_{\text {eq }}$ is 0.4 in $x$-axis when the displacement range of the isolator reaches $\pm 2 \mathrm{~mm}$, which is greater than those for the other two axes. In addition, the values of $\zeta_{\mathrm{eq}}$ in $x$-axis increase with an increase of the displacement range from $\pm 1 \mathrm{~mm}$ to $\pm 2 \mathrm{~mm}$ and then decrease when the displacement range of the isolator is $\pm 3 \mathrm{~mm}$. This axis shows slightly different phenomena than the other two axes; however, the trend is nearly equivalent to the results obtained from Figure 13 of [23].

The estimated equivalent stiffness and damping values of the ring-type SMA mesh washer shown in Figure 6 are much smaller than the results obtained from Figures 5 and 6 of [22], even though their trends are almost all the same. This decrease in the stiffness and damping values is because the density of the SMA mesh washer employed in this study is $50 \%$ less than that used in the previous study. However, once we consider the jitter isolation methodology for the 


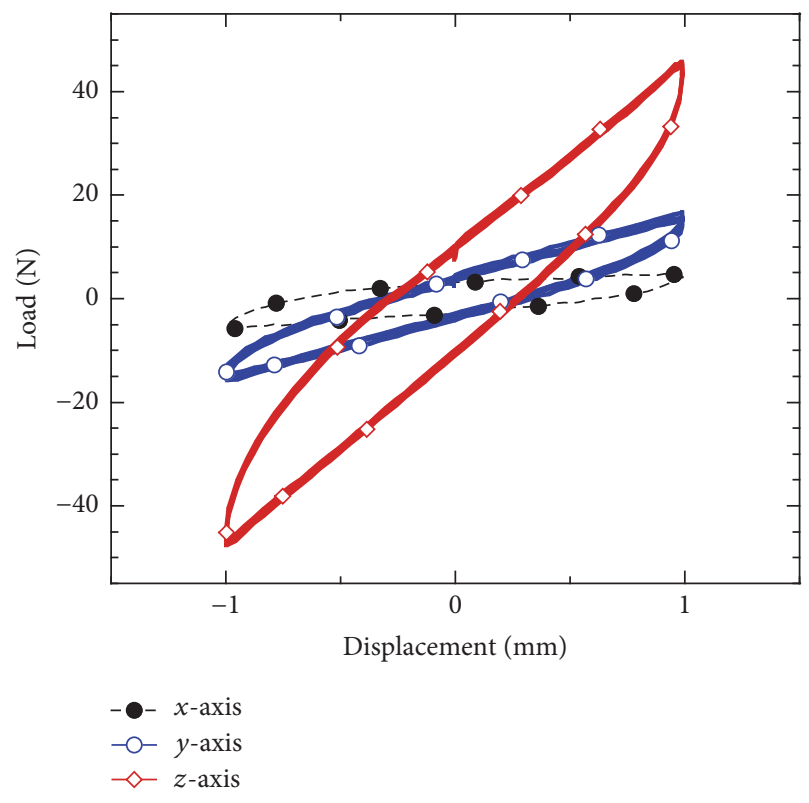

(a) $\pm 1 \mathrm{~mm}$ displacement range

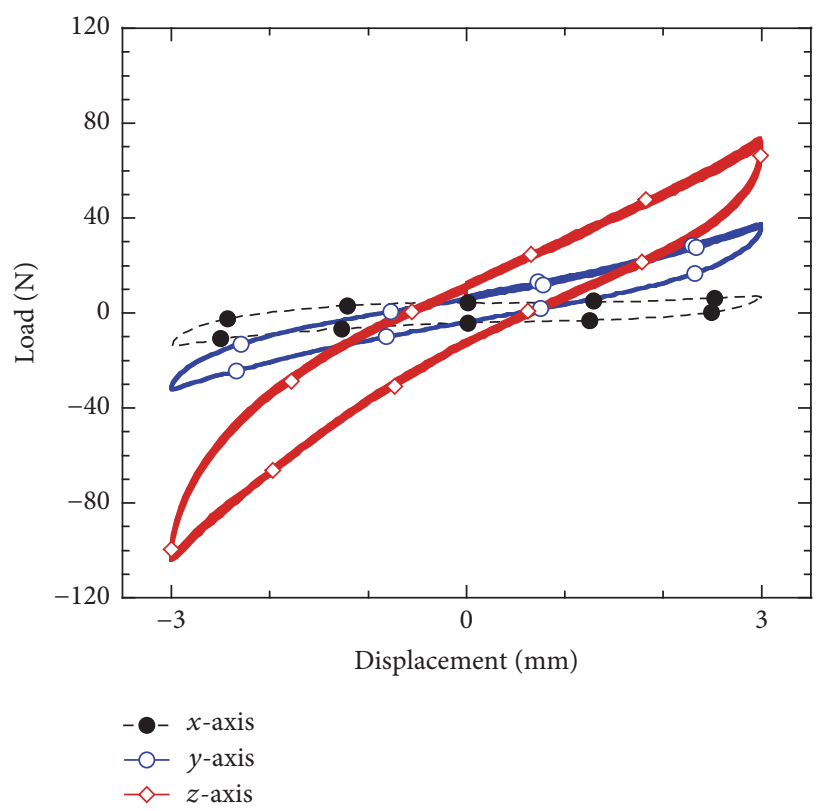

(b) $\pm 3 \mathrm{~mm}$ displacement range

FiguRE 5: Load-displacement relationships for displacement ranges of $1 \mathrm{~mm}$ and $3 \mathrm{~mm}$ on each axis ((a): $x$-axis, (b): $y$-axis, (c): $z$-axis).

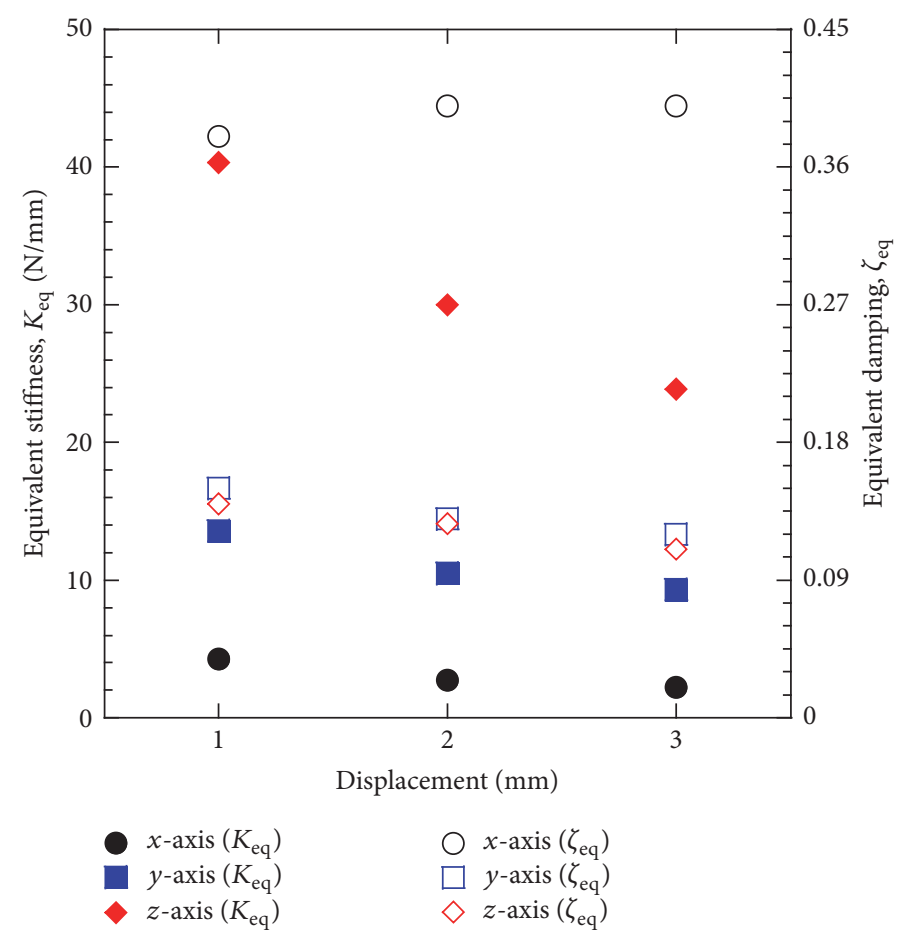

FIGURE 6: Estimated values of the equivalent stiffness $\left(K_{\mathrm{eq}}\right)$ and damping $\left(\zeta_{\mathrm{eq}}\right)$ of the isolator on each axis with respect to displacement ranges.

RWA-induced micro-jitter, a lower stiffness value is always desirable.

2.2. RWA Passive SMA Mesh Washer Isolator. In this study, to verify the effectiveness of the isolator design, which guarantees vibration attenuation in a launch environment while effectively isolating the micro-disturbances in an onorbit environment, RWA for small satellite application was considered as a vibration isolation target for the experimental investigation. The functionality of the RWA considered in this study was verified through the STSAT-2C (Science and Technology Satellite 2C, South Korean Satellite launched in 


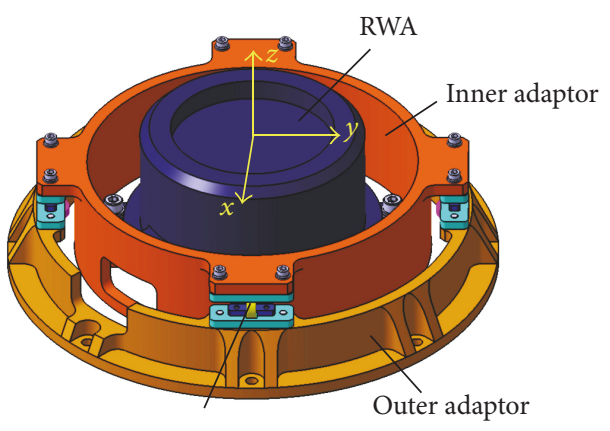

SMA mesh washer isolator (4EA)

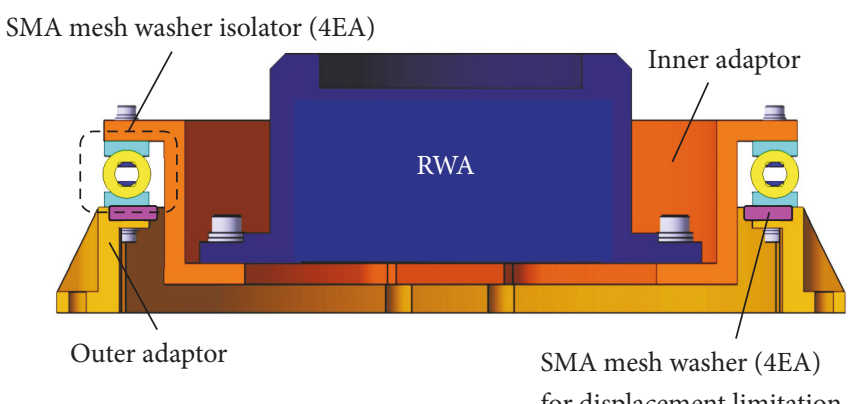

(b)

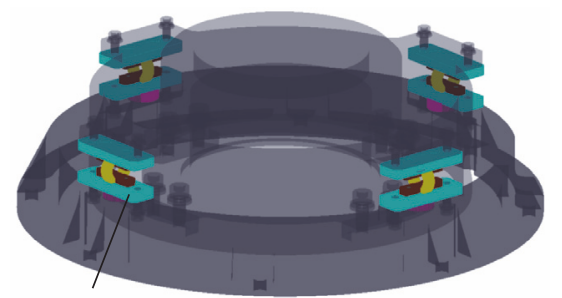

SMA mesh washer isolator (4EA)

(c)

FIGURE 7: Configuration of the RWA combined with the ring-type SMA mesh washer passive isolation system ((a): isometric view, (b): sectional view, and (c): real configuration of the RWA isolation system).

2013) program as the experimental demonstration payload. The mass of the RWA is $1.38 \mathrm{~kg}$; the dimensions are $168 \mathrm{~mm}$ $\times 168 \mathrm{~mm} \times 70 \mathrm{~mm}$; the wheel inertia is $0.001143 \mathrm{kgm}^{2}$; and the maximum reaction torque is $11.1 \mathrm{mNm}$. In this study, a verification model having the same specifications as the RWA flight model was used to verify the isolator proposed in this study.

Figure 7 shows the configuration of a RWA combined with the ring-type SMA mesh washer passive isolation system. To implement the mesh washer isolator onto the RWA, inner and outer adapters were additionally manufactured and integrated into the RWA as shown in Figure 7(a) for the purpose of validation of isolator design as modification of the existing RWA hardware was not allowed anymore.

In case of compatibility of the proposed ring-type SMA mesh washer isolator against different types of RWA, several things should be trade-off studied, that is, the number of ringtype SMA mesh washer isolators and its arrangement with regard to the design requirement of the RWA. Such trade-off study could be carried out based on the basic characteristics of the ring-type SMA mesh washer isolator obtained from static loading tests as shown in Figure 6.

The ring-type isolators are integrated with the RWA through the inner adapter such that they are on the center of the gravity plane of the RWA as shown in Figure 7(b). This allows us to establish a system that does not require an additional holding and release mechanism for the RWA because this greatly minimizes the additional movement of the RWA that is a result of the rotational moments of the RWA. Figure 7(c) shows the accommodation of the SMA mesh washer isolators. The accommodation of the isolators was decided based on the static test results where the RWA isolation system has the 1st eigenfrequency of approximately $18 \mathrm{~Hz}$ in $x-y$ plane for the RWA-induced micro-jitter isolation. Four SMA mesh washers are installed on the outer adapter and are used as a mechanical protector to limit the movements of the RWA within $3.0 \mathrm{~mm}$ in $x-y$ plane under launch vibration environment because the stiffness of the isolator in $x$ - and $y$ axis is much lower than the $z$-axis. This part makes additional contribution of attenuation of the mechanical loads transmitted to the RWA when the inner adapter bumps into outer adapter at around the resonance frequency bands of the RWA isolation system under severe launch environments.

2.3. Numerical Investigation of the RWA Isolation System. Based on the basic characteristic test results of the ring-type SMA mesh washer as shown in Figure 6, it can be noticed that the mechanical behavior of the RWA isolation system varied with regard to the given vibration environments, that is, launch vibration and on-orbit micro-vibration. Under the harsh launch vibration environments, the RWA isolation system experiences large displacements for all axes within $3 \mathrm{~mm}$ due to existence of the mechanical protector. In this condition, once we consider the mechanical property of the ring-type SMA mesh washer and its arrangement in the RWA, the estimated equivalent stiffness of the RWA isolation system along with $x-y$ plane is approximately $24,400 \mathrm{~N} / \mathrm{m}$. Based on this value and the mass of the RWA, the estimated 1st eigenfrequency of the RWA isolation system along $x-y$ plane is $21.1 \mathrm{~Hz}$ under the launch vibration environment. In the same manner, the estimated 1st eigenfrequency of the RWA isolation system along $z$-axis is $40.3 \mathrm{~Hz}$. 


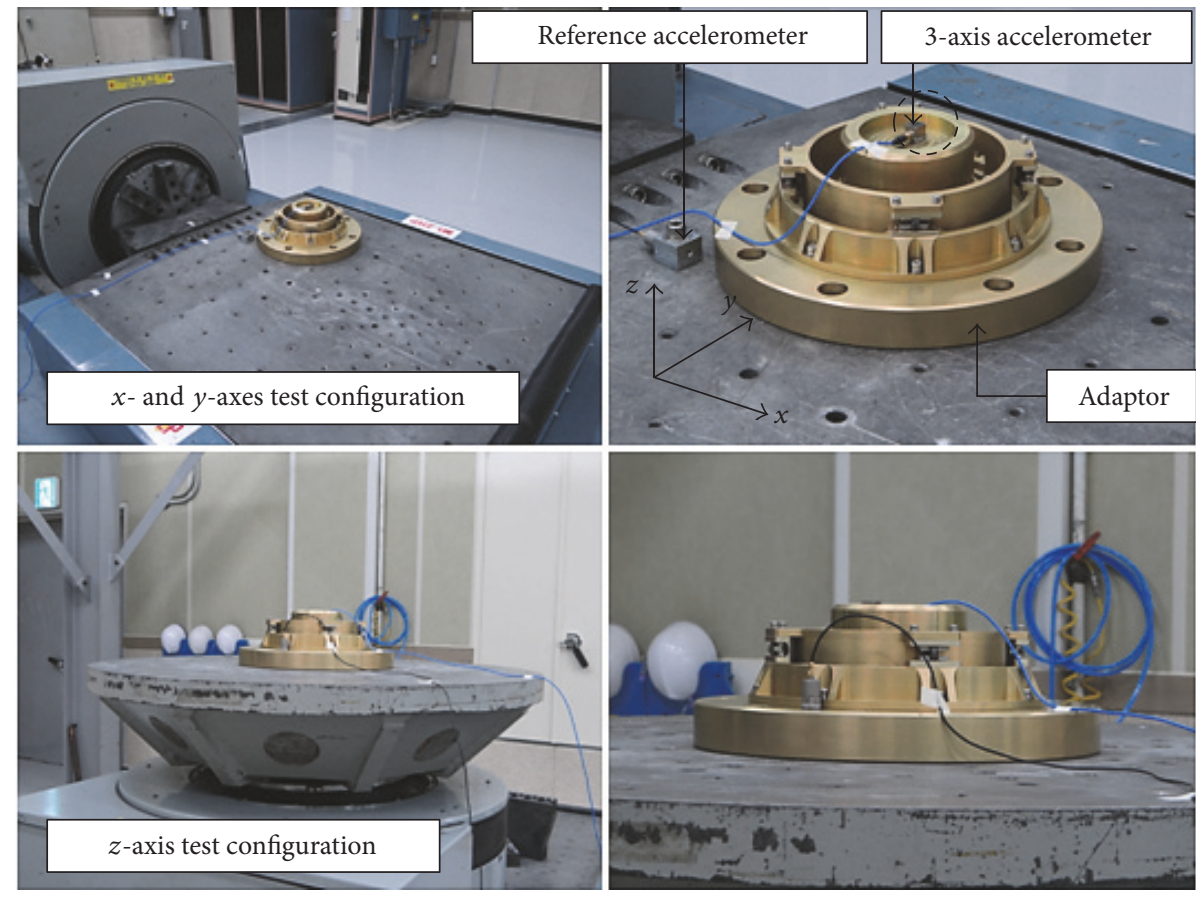

Figure 8: Configuration of the launch vibration test.

On the other hand, under the on-orbit micro-vibration environment, the rotational direction of the RWA is corresponded to the $x$-axis of the ring-type SMA mesh washer. In this condition, the calculated equivalent stiffness of the RWA isolation system, obtained from the case when the ring-type SMA mesh washer is deflected by $1 \mathrm{~mm}$ along $x$ axis, is $17,200 \mathrm{Nm}$. Thus the expected 1st eigenfrequency of the RWA isolation system is $17.76 \mathrm{~Hz}$ under the on-orbit micro-vibration environment. Based on this value, it can be noticed that the RWA isolation system can achieve effective micro-vibration isolation capability through the frequency decoupling methodology.

\section{Experimental Validation of RWA Passive Launch and On-Orbit Isolation System}

3.1. Launch Vibration Test Results. To verify the effectiveness of the isolator design for the launch environment, sine, random vibration tests, and shock tests were performed. In the test, a dummy RWA was used because the test objective was to qualify the isolator design under qualification launch loads. Figure 8 shows the launch vibration test configurations for the $x$-, $y$ - and $z$-axes. The dummy RWA mounted on the isolator had a connection with the vibration shaker through the use of an adaptor. The vibration input from the vibration shaker was obtained from the reference accelerometer. The vibration responses from the RWA isolation system were then obtained from the 3-axis accelerometer placed on the center of gravity of the RWA.

Figures 9(a), 9(b), and 9(c) show the sine vibration test specifications for each axis and the output acceleration measured by the 3-axis accelerometer on the RWA isolation system. In the case of the $x$-axis excitation shown in Figure 9(a), the highest acceleration at the RWA was $7.2 \mathrm{~g}$ at $18 \mathrm{~Hz}$, which was originated from the translational mode of the RWA isolation system along the $x$-axis. This value is almost similar to the estimated 1st eigenfrequency of $21.1 \mathrm{~Hz}$ in Section 2.3. In addition, 2nd highest response was followed by around $23 \mathrm{~Hz}$, which was induced by the rotational mode of the RWA isolation system along the $z$ axis. In the resonance frequency ranges from 18 to $23 \mathrm{~Hz}$, the estimated displacement of the RWA isolation system was $4.3 \mathrm{~mm}$ along the in-plane direction. However, the isolation system cannot exceed $3.0 \mathrm{~mm}$ along the $x$ - or $y$-axis due to the mechanical protector on the outer adapter that limits the movements of RWA within $3.0 \mathrm{~mm}$ as shown in Figure 7(b). Therefore, the inner adaptor bumped into outer adaptor. However, the mechanical vibration loads transmitted to the RWA during the bump between the adaptors were outstandingly dissipated due to the significant damping characteristic of the SMA mesh washer installed on the outer adaptor. This phenomenon contributed towards the reduction of the maximum peak acceleration at the RWA, allowing the design requirement of the RWA to be further mitigated. Alternately, the acceleration responses were greatly reduced after the resonance frequency higher than $23 \mathrm{~Hz}$ because the displacement of the RWA isolation system was reduced below $0.52 \mathrm{~mm}$. Hence, the inner and outer adaptors were no longer bumped into each other. In addition, the frequency decoupling effect due to supporting the RWA with the lowstiffness isolator contributed towards the reduction of the acceleration response for the higher frequency ranges from 24 to $100 \mathrm{~Hz}$. In case of the $y$-axis excitation as shown in Figure 9(b), the highest acceleration at the RWA was $9.2 \mathrm{~g}$ 


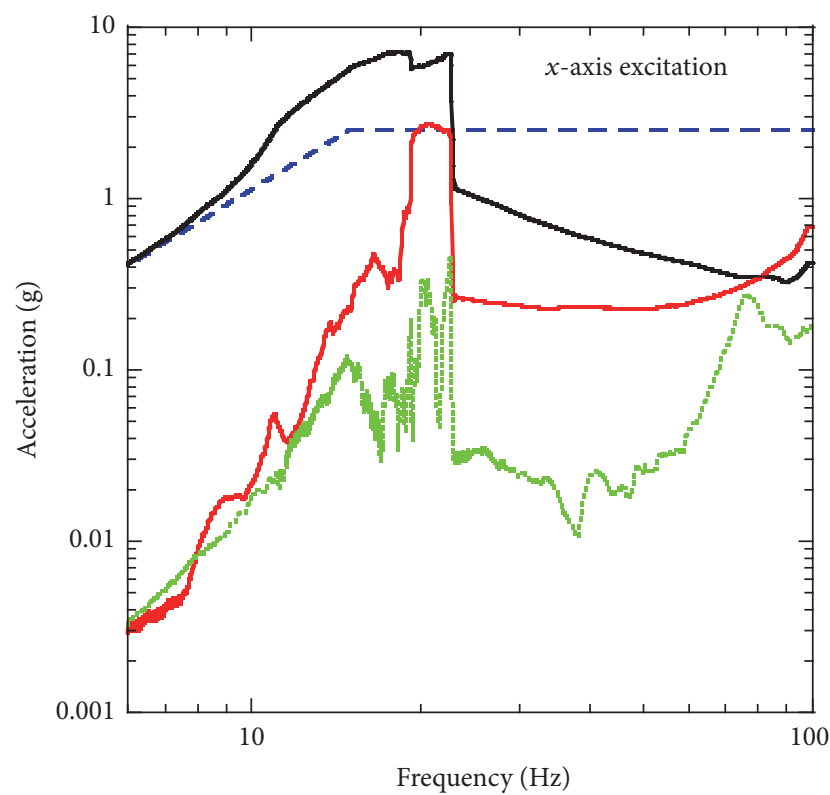

$\begin{array}{lll}-- & \text { Sine test profile } & y(\max .2 .7 \mathrm{~g}) \\ -x(\max .7 .2 \mathrm{~g}) & \cdots . . . & z(\max .0 .4 \mathrm{~g})\end{array}$

(a)

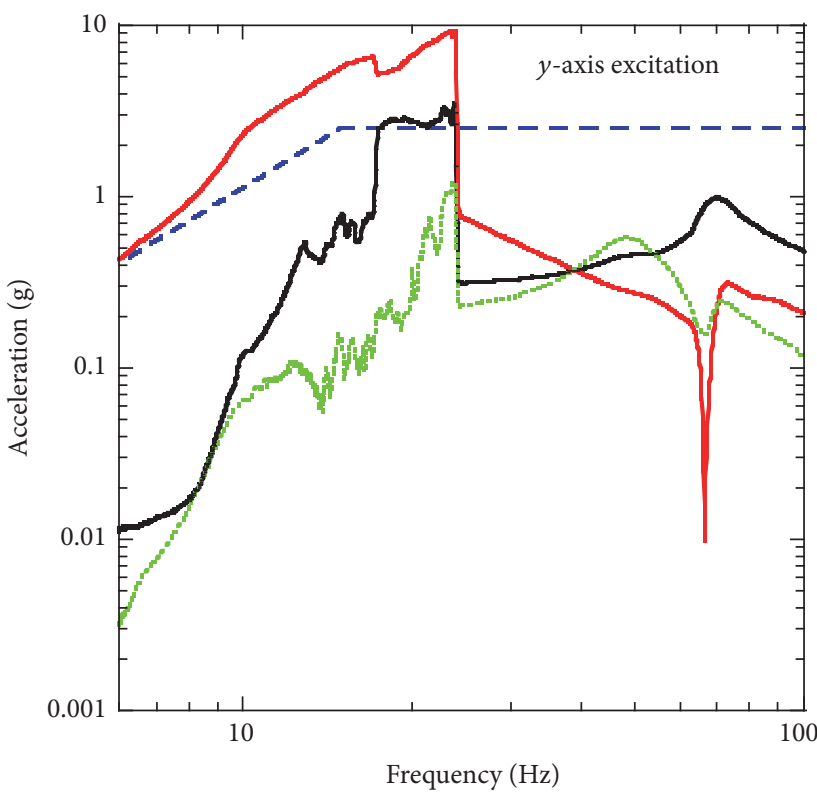

$\begin{array}{lll}--- & \text { Sine test profile } & y(\max .9 .2 \mathrm{~g}) \\ -x(\max .3 .4 \mathrm{~g}) & \ldots . . . & z(\max .1 .2 \mathrm{~g})\end{array}$

(b)

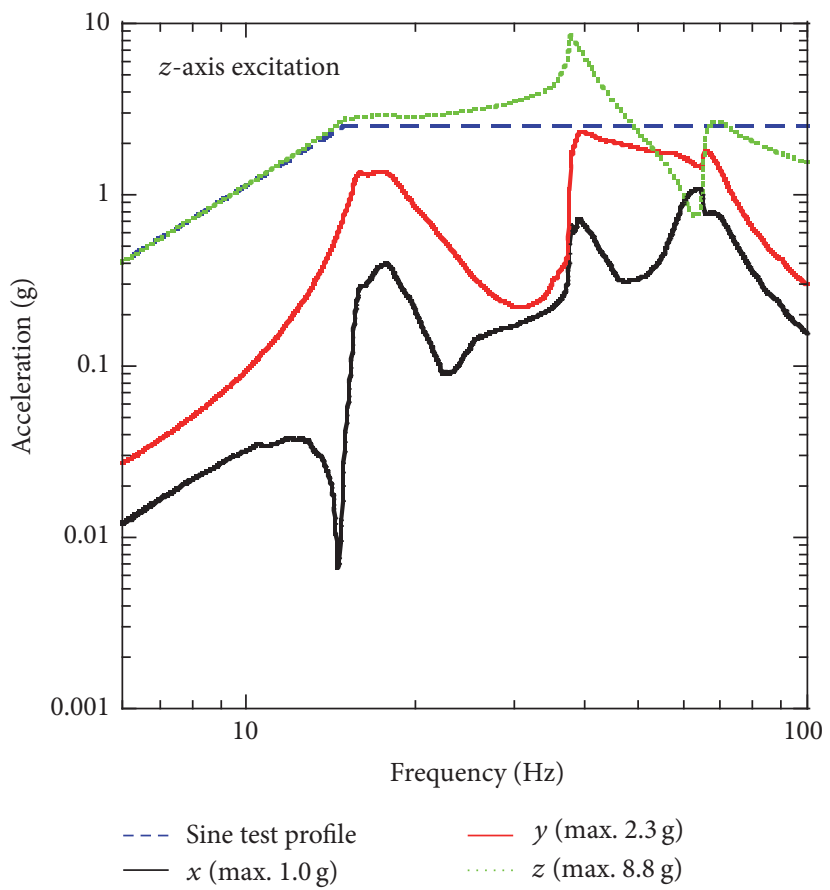

(c)

FIGURE 9: Results for sine vibration tests of the RWA isolation system with respect to each axis excitation ((a): $x$-axis, (b): $y$-axis, and (c): $z$-axis).

at $24 \mathrm{~Hz}$. In addition, the overall tendency showed similar results when compared to the results obtained from the $x$-axis excitation. This was due to the symmetrical design of the SMA mesh washer isolator in the $x-y$ plane as shown in Figure $7(\mathrm{c})$. For the case of the $z$-axis excitation as shown in Figure 9(c), the highest acceleration of $8.8 \mathrm{~g}$ was observed at $38 \mathrm{~Hz}$ which is also almost identical to the estimated 1st eigenfrequency of the RWA isolation system along $z$-axis. In this condition, the estimated deformation of the RWA isolation system along the $z$-axis was $1.5 \mathrm{~mm}$. The resonance frequency observed at $38 \mathrm{~Hz}$ was relatively higher than the 1st eigenfrequency of the RWA isolation system in the $x$ - and $y$-axes, around $18-23 \mathrm{~Hz}$. 
This was because the stiffness of the SMA isolator in the $z$ axis was relatively higher than the $x$ - and $y$-axes, as shown in Figure 5. However, even if the $z$-axis excitation from the vibration shaker is exerted onto the RWA isolation system, a rotational mode of the RWA isolation system along the $x$ and $y$-axes was mainly observed during the vibration test. This rotational mode was identified at approximately $70 \mathrm{~Hz}$, as shown in Figure 9(c). A similar phenomenon can also be observed in the random vibration test results described later. This behavior of the RWA isolation system can be introduced by the fact that the movements of the RWA occurred not only in the $z$-axis but also in the $y-z$ or $x-z$ planes due to accommodations of the SMA mesh washer isolator.

From the above sine vibration test results, it can be seen that the highest acceleration response obtained from each of the $x$-, $y$-, and $z$-axes, when corresponded axis excitation was induced to the RWA isolation system, did not exceed the RWA's design load of $42.1 \mathrm{~g}$, guaranteeing the structural safety of the RWA and isolator itself under sine vibration loads without requiring an additional launch-locking device. However, this design approach cannot be achieved using stainless-steel wire mesh as this material exhibited plastic deformation after a compressive displacement of $0.5 \mathrm{~mm}$ [21]. In addition, because of the relatively higher stiffness value of the steel washer within the elastic range, the jitter isolation capability might be degraded because the isolation capability heavily depends on the stiffness value. For these reasons, the pseudoelastic SMA mesh washer is used in this study.

Figures 10(a), 10(b), and 10(c) show the random vibration test specifications for each axis and the output responses with the same vibration test configuration, which is shown in Figure 8. For comparison of the vibration reduction capabilities of the SMA mesh washer isolator, a random vibration test where the RWA was rigidly mounted onto the adaptor was performed as well. The representative results when the $y$-axis excitation was induced to the RWA without the isolator are shown in Figure 11. In here, the 1st eigenfrequency of the RWA was observed at around $200 \mathrm{~Hz}$, indicating that the vibration loads were amplified to that resonance frequency without isolation and directly transmitted to the RWA. Furthermore, the overshoot at approximately $1000 \mathrm{~Hz}$ was also sharply amplified. This overshoot occurred primarily due to the structural resonance of the mechanical support equipment. The vibration response in the $y$-axis was 175.5 grms. This response level may result in structural problems to the RWA and thereby potentially bring up the structural safety of the RWA during harsh launch vibration environments, especially if the design load of the RWA becomes stricter.

However, the output response in the $y$-axis, when the same axis excitation was induced, was significantly decreased to 0.51 grms by establishing the SMA mesh washer isolator on the RWA as shown in Figure 10(b). Here, it can be noticed that the severe random vibration levels were isolated by a factor of 344 when compared to the rigid condition. This superior vibration isolation capability was due to lowering the 1st eigenfrequency of the RWA from $200 \mathrm{~Hz}$ to $20 \mathrm{~Hz}$. The same vibration isolation methodology was observed in the sine vibration test results, as shown in Figure 9. However, the vibration energy dissipation methodology by a bump between the inner adaptor and the mechanical protector, as experienced during the sine vibration tests, was not seen during the random vibration test as the deformation of the RWA isolation system was too small. Practically, the estimated deformation in the $y$-axis, when the corresponding axis random excitation was induced, was approximately $0.17 \mathrm{~mm}$ at the resonance frequency of $20 \mathrm{~Hz}$. In this condition, the rotational mode of the RWA isolation system along the $x$-axis was also observed at approximately $70 \mathrm{~Hz}$. On the other hand, a similar behavior of the RWA isolation system in the $x$-axis was seen in the overall frequency bands when the $x$-axis excitation was induced. This behavior was due to the symmetric configurations of the RWA isolation system in the $x-y$ plane.

The response along the $z$-axis, when the $z$-axis excitation was induced to the RWA isolation system, indicated slightly different results compared to the $x$ - or $y$-axis responses. Because the stiffness of the SMA mesh washer isolator in the $z$-axis was relatively higher than those of the $x$-and $y$-axis, therefore, the 1st eigenfrequency of the RWA isolation system along the $z$-axis was observed at around $30 \mathrm{~Hz}$. In contrast, as indicated in basic characteristic results, the equivalent damping in the $z$-axis was relatively lower than for the other axes. Therefore, the peak occurring at 1st eigenfrequency was relatively higher than the other axes. However, this was no longer of concern in regard to the structural safety of the RWA because the estimated acceleration response at the resonance frequency did not exceed the design load of the RWA. Additionally, the random vibration input level of 14.1 grms was significantly decreased to 2.89 grms.

Figure 12 shows the representative frequency response spectrum of the RWA isolation system obtained from the low level random sweep (LLRS) tests along the $y$-axis before and after the launch vibration tests referred to above. This test was performed to investigate the structural safety of the RWA isolation system by comparing the dynamic response of the RWA isolation system before and after the launch vibration tests. In general, to satisfy the test requirement of the vibration test, no variations of 1st eigenfrequency less than $5 \%$ and amplitudes less than $3 \mathrm{~dB}$ should be observed from the LLRS test. The results of LLRS indicate that the 1st eigenfrequency of the RWA isolation system along the $y$-axis was observed at around $18-20 \mathrm{~Hz}$, which is the same as the results obtained from the launch vibration tests. This result also indicated that no variations of frequencies and amplitudes before and after the launch vibration tests occurred. Hence, the structural safety of the isolator itself can be guaranteed in spite of being subjected to harsh launch vibration loads. Therefore, it is expected that no performance degradation for the microjitter isolation in the on-orbit environment occurs.

From this test result, it can be expected that a significantly deformed SMA mesh washer, occurring in a launch environment, can recover its original shape without plastic deformation upon unloading in a 0 -g environment owing to its pseudoelastic behavior, as shown in the static test results. These facts are great advantages to use the pseudoelastic SMA mesh washer in this study as a smart adaptive system that can be used in both a launch environment and on-orbit microjitter isolation without requiring an additional launch lock device. 


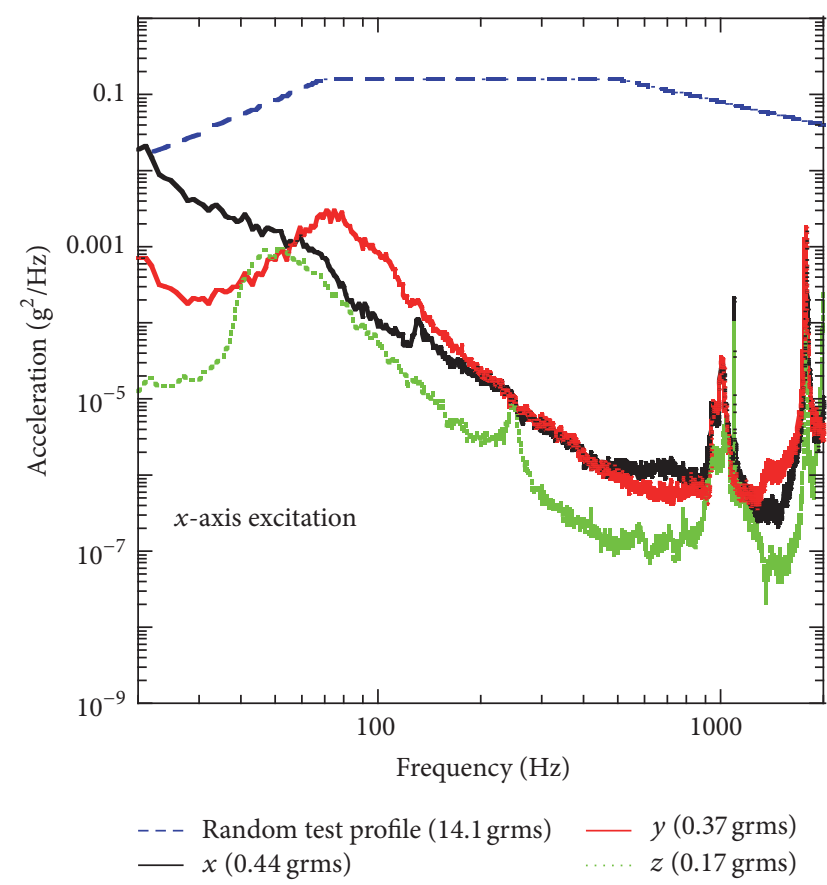

(a)

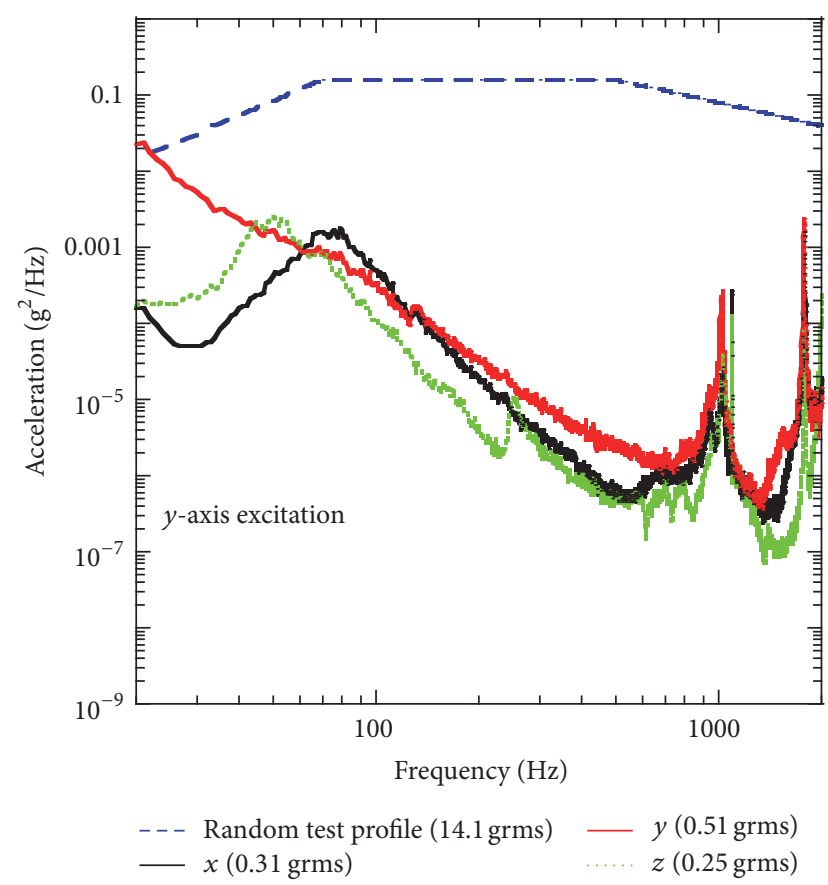

(b)

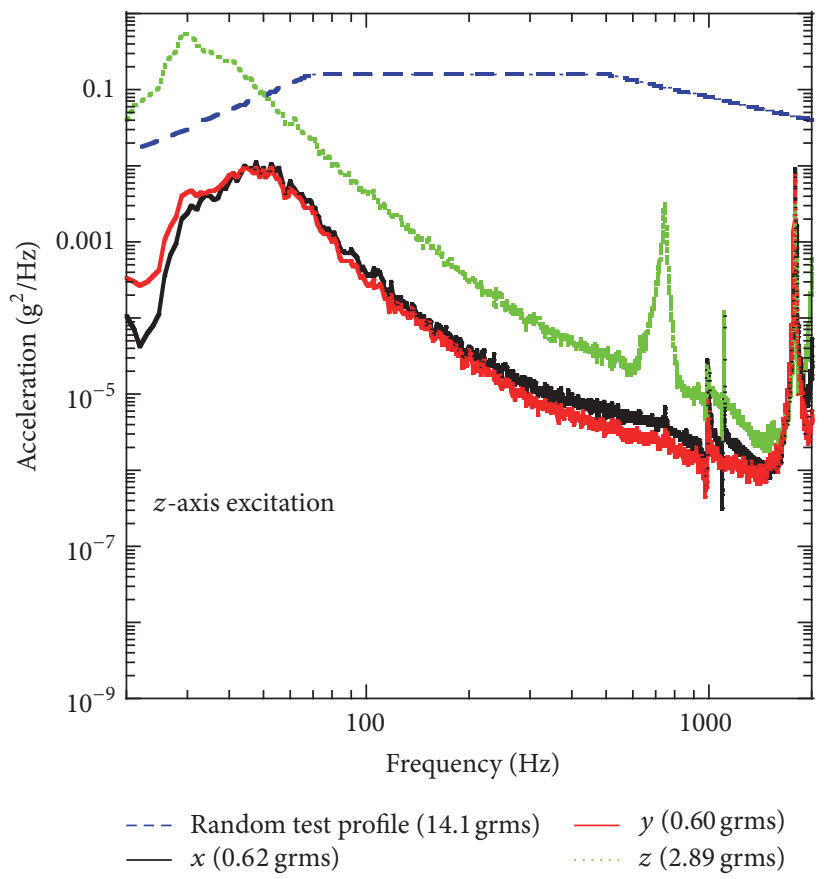

(c)

FIGURE 10: Results for random vibration tests of the RWA isolation system with respect to each axis excitation ((a): $x$-axis, (b): $y$-axis, and (c): $z$-axis).

On the other hand, the ring-type SMA mesh washer is subjected to substantial cyclic deformations during launch vibration environments; thus a functional fatigue such as degradations of superelasticity or shape recovery effect can be a concern. In general, the formation and growth of microscopic defects such as dislocations of the superelastic SMA material are caused by cyclic loadings. These dislocations are formed and aggregated mainly near the interfaces between austenite and stress-induced martensite phases and the interfaces between the martensite variants with different crystallographic orientations [24]. Such dislocations in the SMA material result in occurrence of residual strain in the SMA itself and the residual strain gradually increases as the loading cycle increases. Besides, the hysteresis area becomes lower as 


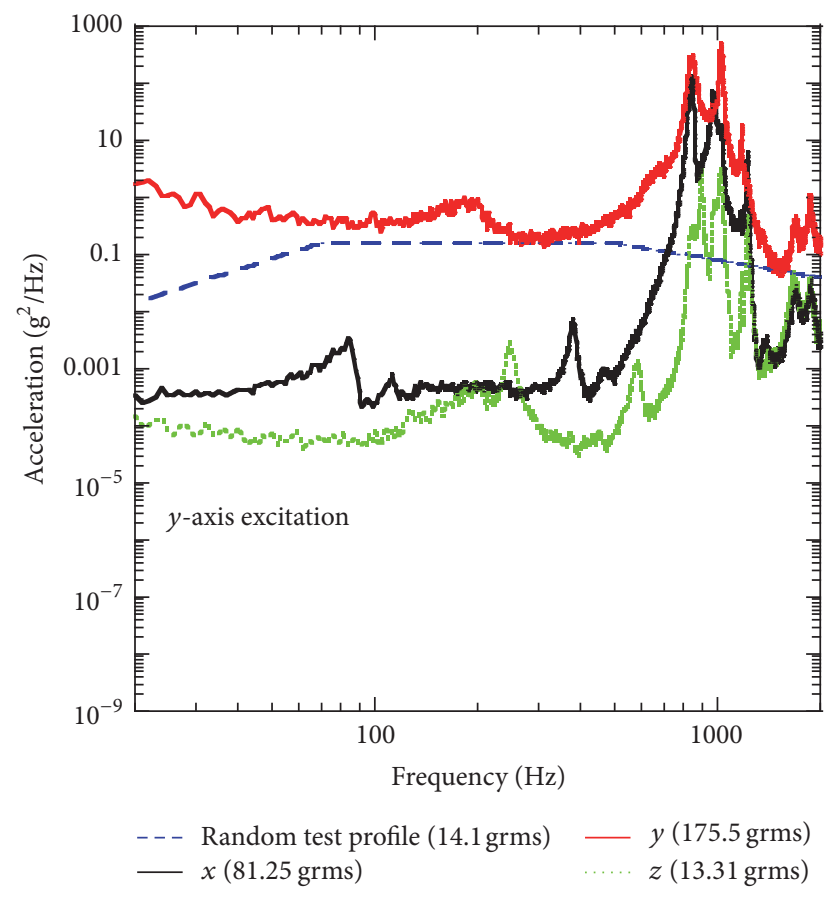

FIGURE 11: Representative results for random vibration tests of the RWA without isolation system when the $y$-axis excitation was induced.

the loading cycle increases [25]. This means that the damping capability of the superelastic SMA is decreased if the cyclic loadings are repeatedly accumulated in the SMA material. However, once we investigate the static loading test result for $\pm 3 \mathrm{~mm}$ as shown in Figure 5(b), no phase transformation phenomenon from austenite to martensite can be observed under given displacement limits of $\pm 3 \mathrm{~mm}$ which is the maximum allowable deflection of the RWA isolation system due to a mechanical protector. Therefore, considering the main fatigue failure mechanism of the superelastic SMA such as the interfaces between austenite and stress-induced martensite phases, it can be revealed that no severe failure mechanism acts on the ring-type SMA mesh washer during launch vibration environments. Moreover, it can be also noticed from the LLRS test result, shown in Figure 12, that no variations of frequencies and amplitudes before and after the launch vibration tests occurred. Hence, the fatigue effect can be negligible to the current design of the RWA isolation system proposed in this study.

Figure 13 shows the shock test results for the $x$-, $y$-, and $z$-axes when each shock excitation along the corresponding axis was induced. The maximum shock response spectrum (SRS) on the RWA based on a maximum SRS input of $793 \mathrm{~g}$ was $3.3 \mathrm{~g}$ in the $y$-axis, thereby indicating that the shock level transmitted to the RWA was greatly attenuated by the factor of 240 . This superior attenuation capability was due to the frequency decoupling principal that occurred when mounting the RWA with a low-stiffness isolator. The 1st eigenfrequency of the RWA isolation system in the $x$ and $y$-axes was observed at around $20 \mathrm{~Hz}$. This was similar when compared with the results from the sine and random vibration tests for the identical axis, including the $z$-axis responses.

These test results indicate that the isolation system using the pseudoelasticity of the SMA mesh washer is effective for guaranteeing the structural safety of a RWA without requiring an additional launch-locking device and greatly reducing the harsh vibration loads transmitted to the RWA that enables mitigating the strict design requirements of the RWA.

3.2. Micro-Jitter Measurement Test Results. Generally, it has been known that micro-jitter disturbances induced by the RWA activation can be divided into three categories based on their origin: rotor imbalance, ball bearing imperfection, and motor driven disturbances [12]. In here, it has also been known that the rotor imbalance of the RWA has the most significant effect on micro-jitter disturbances and generates disturbances at the same frequency of rotation, which is referred to as fundamental harmonic (H1). At some wheel speed, this fundamental harmonic can be significantly amplified through frequency coupling with the structural modes of the RWA. This effect will seriously impact the image quality of a high-resolution observation satellite. Alternately, irregularities in ball bearings, motor, lubrication, and so forth generate disturbances that usually occur at integral and fractional multiples of the fundamental harmonic frequency as sub- and superharmonics ( $\mathrm{H} 0.5, \mathrm{H} 2, \mathrm{H} 2.7, \mathrm{H} 3$, etc.). However, these disturbances from the irregularities are usually significantly smaller than those of the fundamental harmonics.

To investigate the micro-jitter isolation performance when employing a new application of the SMA mesh washer as proposed in this study, a micro-jitter measurement test, with and without an isolation system, was performed on the Kistler table. Figure 14 shows the micro-jitter measurement test set-up with and without the RWA isolation system. The RWA was installed on the Kistler table through inner and outer adaptor, and four isolators bridged the inner and outer adaptor with a low stiffness, respectively. The test set-up without the isolation system was implemented by rigidly connecting those inner and outer adaptor, such that the microjitter from the RWA was directly transmitted to the Kistler table without isolation. In the micro-vibration measurement test of the RWA, the $0 \mathrm{~g}$ device compensating for the $1 \mathrm{~g}$ effect during the ground test was not applied to simulate zero gravity on-orbit environment. Therefore, the mesh washer was subjected to prestressed conditions owing to the RWA mass, including the inner adaptor for a total of $2 \mathrm{~kg}$. In this condition, the estimated deflection of the RWA isolation system along the direction of gravity due to the RWA with an adaptor was $0.12 \mathrm{~mm}$. However, the estimated deflection might not have a considerable effect on the eigenfrequency shift of the isolation system between $1 \mathrm{~g}$ and $0 \mathrm{~g}$ conditions because the stiffness variation of the mesh washer due to RWA mass was negligibly small. During the test, the free-rundown method, which decreased the wheel speed naturally by turning off the operation command, was adopted to investigate the impact of the micro-jitter of the RWA, such that the wheel speed of the RWA was varied from 5000 to $0 \mathrm{rpm}$ in the test.

Figures 15(a) and 15(b) show examples of waterfall plots of the RWA disturbance forces and torques without the isolation 


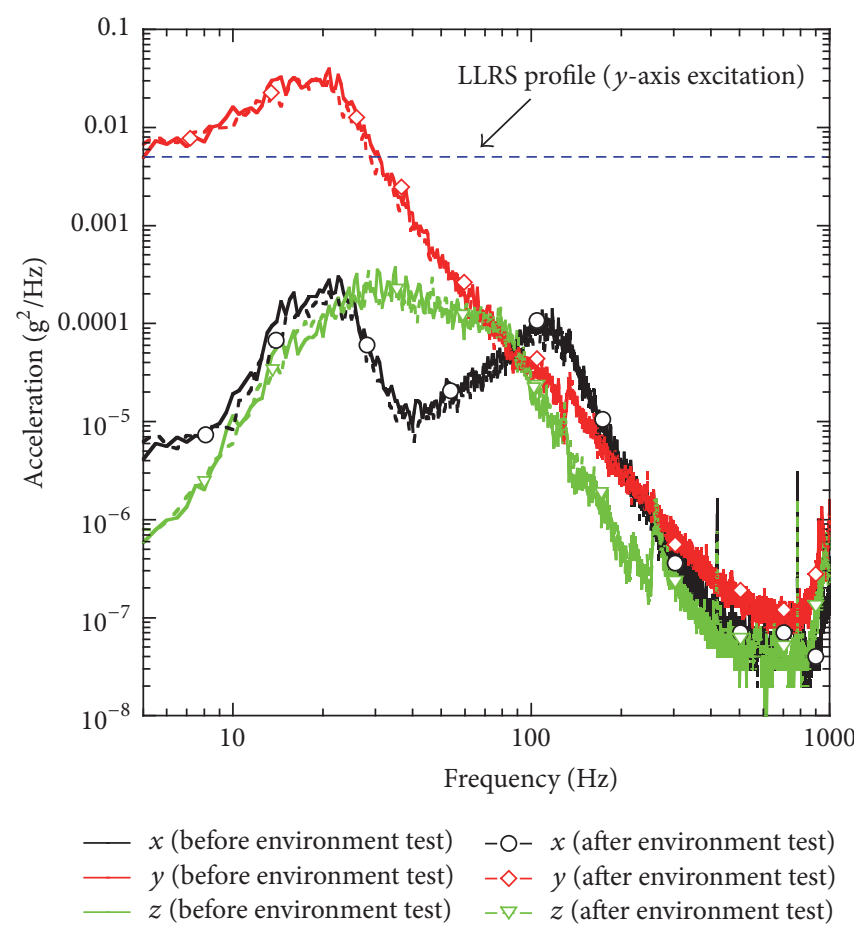

FIGURE 12: Frequency response spectrum of the RWA isolation system obtained from the low level random sweep (LLRS) test on the $y$-axis before and after the launch vibration tests.

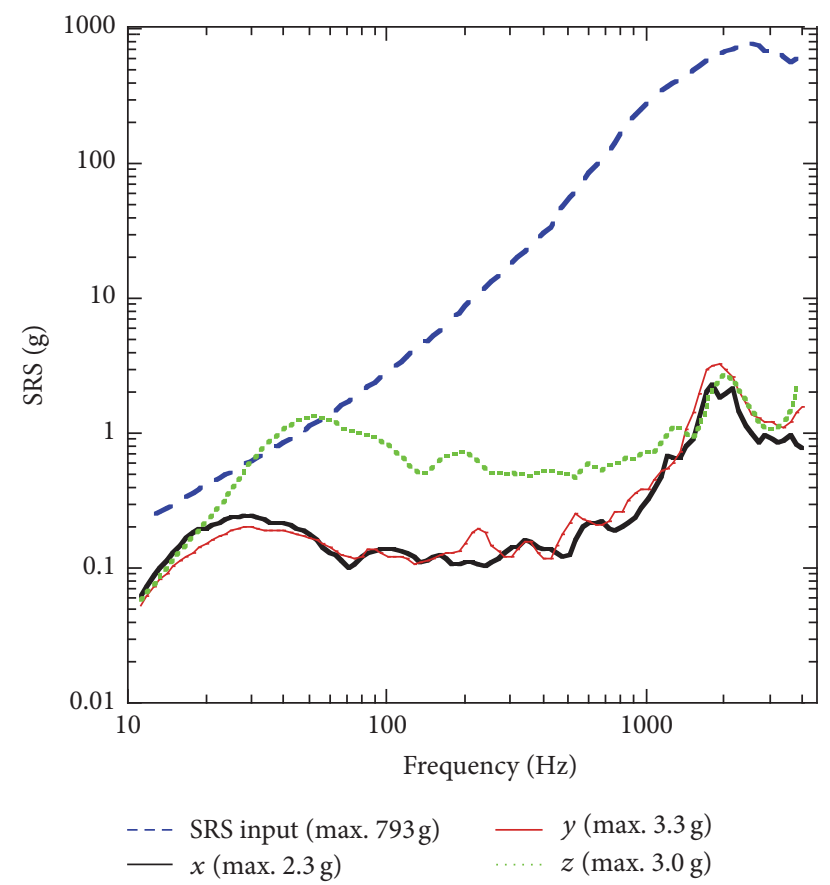

FIGURE 13: Results for shock test of RWA isolation system on each axis excitation.

system. From these results, it can be seen that the fundamental harmonic at the $5000 \mathrm{rpm}$ has the largest amplitude and gradually decreases as the wheel speed decreases. In here, the amplitude of the fundamental harmonic is proportional to the square of the wheel speed [26]. Aside from the fundamental harmonic, a number of superharmonics were also observed in all axes with relatively lower amplitudes compared to the fundamental harmonic.

The disturbance, which appears at frequency of $130 \mathrm{~Hz}$ with same amplitude throughout the entire test speed ranges, mainly comes from the electrical power supply equipment. In practice, this electrical noise cannot be avoidable during the test even if some efforts were implemented towards reducing this noise [26]. However, the electrical noise is much smaller when compared to the harmonic disturbances; therefore, it can be ignored. Meanwhile, the peak disturbances occurring at around $280 \mathrm{~Hz}$ and $420 \mathrm{~Hz}$ primarily result from the frequency coupling effect between the RWA structural modes and the harmonic disturbances. These peak disturbances were observed in all axes.

Figures 16(a) and 16(b) show waterfall plots of the RWA disturbance forces and torques with the isolation system. The test was conducted under the same conditions as the without isolation test to impartially assess the micro-jitter isolation performance of the RWA isolation system proposed in this study. From this result, it can be seen that the fundamental harmonic has a different shape and its maximum amplitudes in all waterfall plots are considerably reduced when compared to the results of the rigid condition. For example, in the case of the $x$-axis, the maximum peak value occurring at the fundamental harmonic without the isolation is approximately $3.9 \mathrm{~N}$ at $80 \mathrm{~Hz}$, which corresponds to a maximum wheel speed of 5000 RPM. However, this value is effectively attenuated to $1.8 \mathrm{~N}$ by the factor of 2.16 at the same wheel speed condition due to the SMA isolation system. In fact, the main feature of 

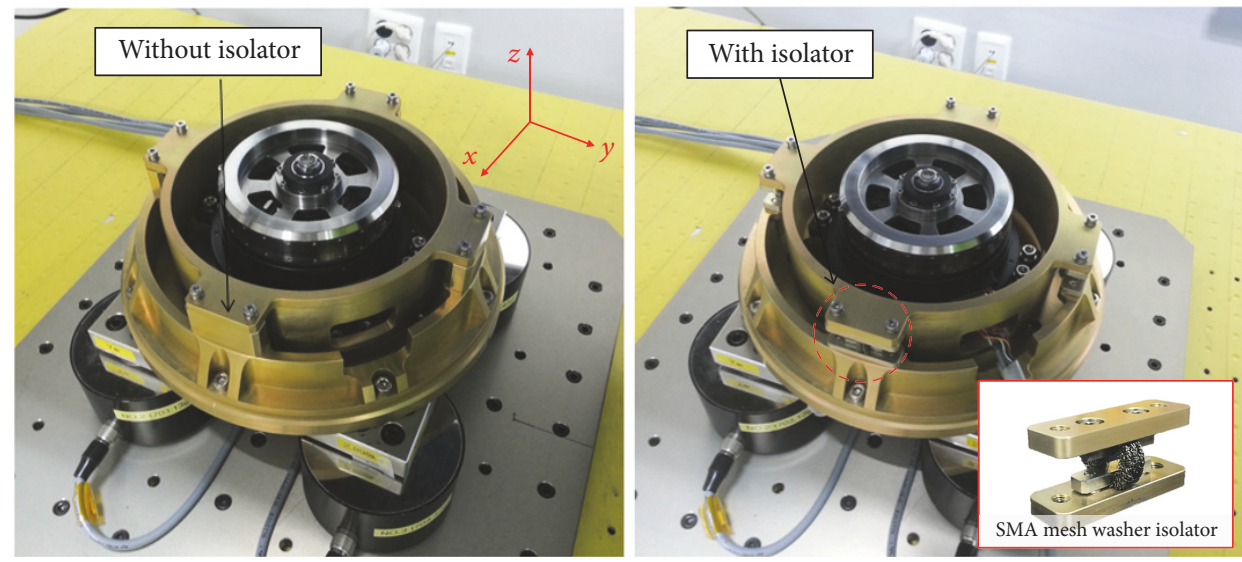

FIGURE 14: Test set-up for micro-jitter measurement of RWA with and without isolation system.
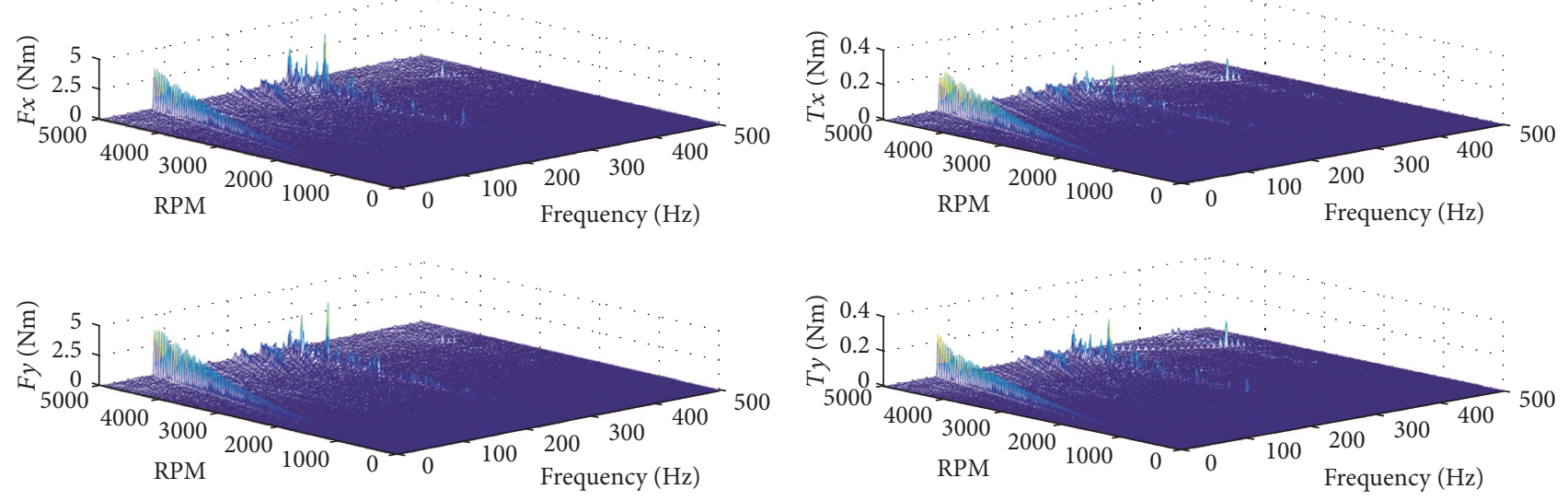

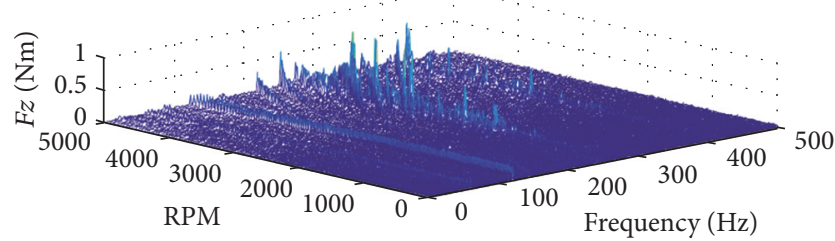

(a)

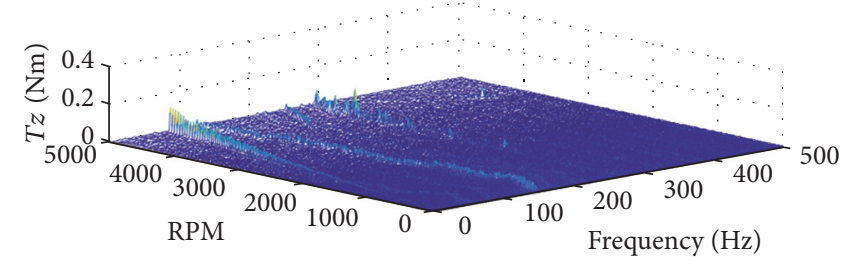

(b)

FIGURE 15: Waterfall plots of disturbance forces and torques for RWA activation without isolation ((a): disturbance forces and (b): disturbance torques).

the RWA-induced micro-jitter isolation, when utilizing the proposed application form of SMA mesh washer, is to put the eigenfrequency of the isolation system towards a lower frequency region than that of the without isolation condition to effectively reduce higher frequency disturbances. The 1st eigenfrequency of the RWA with the isolation system is observed in a low-frequency range at around $20 \mathrm{~Hz}$ in the $x$ - and $y$-axes, which is the same as the results obtained from the launch vibration tests. These frequencies are much lower than the eigenfrequency of a RWA without isolation system, which is roughly $280 \mathrm{~Hz}$. Therefore, the harmonic disturbances induced by the RWA activation as well as the structural modes of the RWA itself are effectively isolated on the basis of the 1st eigenfrequency of the isolation system.
However, some amplitudes are still observed in the waterfall plots because the fundamental harmonic of the RWA and the 1st eigenfrequency of the isolation system are coupled with each other at a specific wheel speed region, which corresponds to approximately 1700 RPM. These peaks are not avoidable in the current system because the isolation methodology proposed in this study is based on the passive approach of frequency decoupling. However, the maximum peak force and torque level at the resonance frequency of isolation system, $20 \mathrm{~Hz}$, are also effectively attenuated due to the significant damping characteristics of the SMA mesh washer as shown in Figure 6.

On the other hand, the 1st eigenfrequency of the RWA isolation system along the $z$-axis is not clearly observed in 

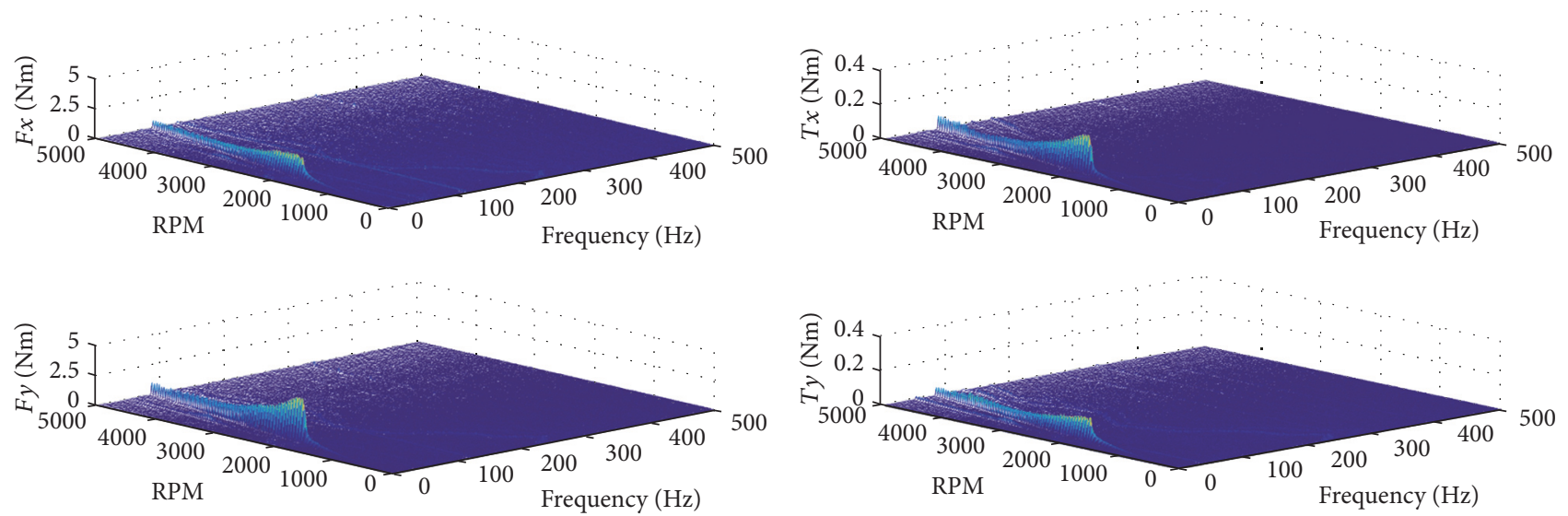

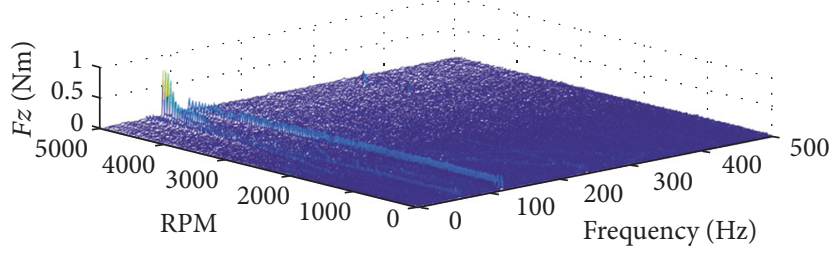

(a)

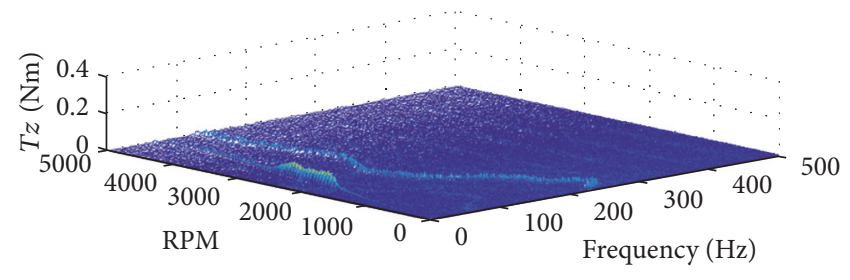

(b)

FIGURE 16: Waterfall plots of disturbance forces and torques for RWA activation with isolation ((a): disturbance forces and (b): disturbance torques).

the waterfall plot because the main jitter contributor of the RWA is the rotational movement in the $x-y$ plane. Therefore, the micro-jitter disturbances along the $z$-axis are less critical when compared to the $x$ - or $y$-axis. In practice, the maximum peak value with the isolation system in the $z$-axis is approximately $0.3 \mathrm{~N}$ at the maximum wheel speed and this value is almost negligible. However, from the basic characteristic and launch vibration tests results, it can be assumed that it existed at around $40 \mathrm{~Hz}$ and this value is included in the fundamental harmonic frequency ranges of the RWA. Therefore, the fundamental harmonic disturbances are slightly amplified when coupled with the 1st eigenfrequency of the isolation system along the $z$-axis. However, higher harmonics including structural modes of the RWA are significantly isolated due to the frequency decoupling. For instance, the maximum peak value of $1.7 \mathrm{~N}$ at $280 \mathrm{~Hz}$ without isolation system decreases to nearly $0 \mathrm{~N}$ with the isolation system.

In terms of position sensitivity of the ring-type SMA mesh washer on the micro-jitter isolation performance, the equivalent stiffness and damping values along $x$ - and $y$-axes, which are corresponded to the main excitation axis of the RWA, are not significantly varied as the displacement changes, as shown in Figure 6. This means that the dynamic characteristic of the RWA isolation system does not significantly change with regard to the alignment shift of the ring-type SMA mesh washer. Thus, stable micro-jitter isolation performance can be expected regardless of position sensitivity.

For a more detailed investigation with regard to the effectiveness of the isolation system, the total disturbances reduction ratio obtained with and without the isolation system is calculated and summarized in Table 1 . This table shows the ratio of maximum and mean peak reduction and the standard deviation reduction for each axis. From the results, it can be noticed that the maximum peak reduction ratios of $59.32 \%$ and $42.92 \%$ for forces and torques are observed on the $F x$ and $T y$, respectively. The $F x$ result also shows the highest peak mean reduction ratio of $58.94 \%$ while the highest value of $53.41 \%$ for torque is obtained from $T y$. With regard to the standard deviation reduction ratio for forces and torques, all axes showed considerable reduction ratios compared to the rigid condition. This reduction occurred because the high frequency disturbances were all significantly isolated due to the implementation of the isolation system. In here, the main contribution of micro-jitter isolation mainly results not from the pseudoelastic behavior of SMA effects but from the relatively lower stiffness of the isolator. The microjitter measurement test results indicate that the application of the SMA mesh washer isolator is effective in reducing RWA-induced micro-jitter when mounting the RWA with a low rotational stiffness isolator, which is identical to the main jitter sources induced by the rotational movement of the RWA. The SMA isolator proposed in this study is also effective in the $z$-axis even though its stiffness is relatively higher than those for the $x$ - and $y$-axis.

\section{Conclusion}

In this study, a novel application form of the ring-type SMA mesh washer isolator that can be used in both launch vibration and on-orbit micro-jitter environments without requiring a launch lock device was evaluated. The RWA for a small satellite application was considered as a vibration isolation target for the purpose of experimentally investigating the SMA mesh washer isolator as proposed in this study. The 
TABLE 1: Total disturbance reduction ratio, with and without isolators.

\begin{tabular}{lcccccc}
\hline Reduction rate & $F_{x}$ & $F_{y}$ & $F_{z}$ & $T_{x}$ & $T_{y}$ & $T_{z}$ \\
\hline Peak reduction (\%) & & & & & & \\
$\quad$ Max. & 59.32 & 23.65 & 44.37 & -0.04 & 42.93 & 30.12 \\
$\quad$ Mean & 58.944 & 37.01 & 55.64 & 42.72 & 53.41 & 19.49 \\
STD reduction (\%) & 52.05 & 22.49 & 58.71 & 15.34 & 42.41 & 32.92 \\
\hline
\end{tabular}

basic characteristics of the SMA mesh washer isolator were demonstrated through the static loading tests.

The effectiveness of the isolator design in launch environments was assessed through qualification level sine, random vibration, and shock tests. The results of the LLRS before and after the launch vibration tests indicated that the structural safety of the RWA isolation system subjected to harsh vibration loads was guaranteed without any variations of frequencies and amplitudes. The micro-jitter measurement test results indicated that the RWA-induced micro-jitter harmonics including RWA structural modes were outstandingly isolated due to shifting the 1st eigenfrequency of the RWA isolation system towards a lower frequency.

\section{Conflicts of Interest}

The authors declare that there are no conflicts of interest regarding the publication of this paper.

\section{Acknowledgments}

This research was supported by National Research Foundation of Korea (NRF) funded by the Ministry of Science, ICT and Future Planning (MSIP) (NRF-2015R1A2A2A01003672).

\section{References}

[1] K. KOMATSU and H. UCHIDA, "Microvibration in spacecraft," Mechanical Engineering Reviews, vol. 1, no. 2, pp. SE0010SE0010, 2014.

[2] X. Li and W. Cheng, "Test and analysis of micro-vibrations generated by large control moment gyroscope," International Journal of Control and Automation, vol. 7, no. 2, pp. 297-308, 2014.

[3] S. Riabzev, A. Veprik, H. Vilenchik, and N. Pundak, "Control of dynamic disturbances produced by a pulse tube refrigerator in a vibration-sensitive instrumentation," Cryogenics, vol. 49, no. 1 , pp. 7-11, 2009.

[4] D. K. Kim and H. T. Choi, "Velocity optimization method of Xband antenna for jitter attenuation," in Proceedings of the The 21st International Congress on Sound and Vibration, Beijing, China, 2014.

[5] Z. Zhang, G. S. Aglietti, and W. Zhou, "Microvibrations induced by a cantilevered wheel assembly with a soft-suspension system," AIAA Journal, vol. 49, no. 5, pp. 1067-1079, 2011.

[6] D. Kamesh, R. Pandiyan, and A. Ghosal, "Passive vibration isolation of reaction wheel disturbances using a low frequency flexible space platform," Journal of Sound \& Vibration, vol. 331, no. 6, pp. 1310-1330, 2012.

[7] P. Davis, D. Cunningham, and J. Harrell, "Advanced $1.5 \mathrm{~Hz}$ passive viscous isolation system," in Proceedings of the 35th
AIAA/ASME/ASCE/AHS/ASC Structures, Structural Dynamics, and Materials Conference, Hilton Head, SC, USA, 1994.

[8] Y. Zhang, Z. Guo, H. He, J. Zhang, M. Liu, and Z. Zhou, "A novel vibration isolation system for reaction wheel on space telescopes," Acta Astronautica, vol. 102, pp. 1-13, 2014.

[9] H.-U. Oh, K. Izawa, and S. Taniwaki, "Development of variabledamping isolator using bio-metal fiber for reaction wheel vibration isolation," Smart Materials and Structures, vol. 14, no. 5, pp. 928-933, 2005.

[10] H.-U. Oh, S. Taniwaki, N. Kinjyo, and K. Izawa, "Flywheel vibration isolation test using a variable-damping isolator," Smart Materials and Structures, vol. 15, no. 2, pp. 365-370, 2006.

[11] K. J. Pendergast and C. J. Schauwecker, "Use of a passive reaction wheel jitter isolation system to meet the Advanced Xray Astrophysics Facility imaging performance requirements," in Proceedings of the 1998 Conference on Space Telescopes and Instruments V. Part 1 (of 2), pp. 1078-1094, March 1998.

[12] Z. Zhang, G. S. Aglietti, W. Ren, and D. Addari, "Microvibration analysis of a cantilever configured reaction wheel assembly," Advances in Aircraft and Spacecraft Science, vol. 1, no. 4, pp. 379398, 2014.

[13] W.-Y. Zhou, G. S. Aglietti, and Z. Zhang, "Modelling and testing of a soft suspension design for a reaction/momentum wheel assembly," Journal of Sound and Vibration, vol. 330, no. 18-19, pp. 4596-4610, 2011.

[14] L. P. Davis, J. F. Wilson, R. E. Jewell, and J. J. Roden, Hubble Space Telescope Reaction Wheel Assembly Vibration Isolation System, NASA Marshall Space Flight Centre, 1986.

[15] H.-U. Oh, K.-J. Lee, and M.-S. Jo, "A passive launch and onorbit vibration isolation system for the spaceborne cryocooler," Aerospace Science and Technology, vol. 28, no. 1, pp. 324-331, 2013.

[16] H.-U. Oh, S.-C. Kwon, and S.-H. Youn, "Characteristics of spaceborne cooler passive vibration isolator by using a compressed shape memory alloy mesh washer," Smart Materials and Structures, vol. 24, no. 1, Article ID 015009, 2015.

[17] S.-C. Kwon, S.-H. Jeon, and H.-U. Oh, "Performance evaluation of spaceborne cryocooler micro-vibration isolation system employing pseudoelastic SMA mesh washer," Cryogenics, vol. 67, pp. 19-27, 2015.

[18] S.-H. Youn, Y.-S. Jang, and J.-H. Han, "Compressed mesh washer isolators using the pseudoelasticity of SMA for pyroshock attenuation," Journal of Intelligent Material Systems and Structures, vol. 21, no. 4, pp. 407-421, 2010.

[19] H.-K. Jeong, J.-H. Han, S.-H. Youn, and J. Lee, "Frequency tunable vibration and shock isolator using shape memory alloy wire actuator," Journal of Intelligent Material Systems and Structures, vol. 25, no. 7, pp. 908-919, 2014.

[20] S.-H. Youn, Y.-S. Jang, and J.-H. Han, "Development of a threeaxis hybrid mesh isolator using the pseudoelasticity of a shape memory alloy," Smart Materials and Structures, vol. 20, no. 7, Article ID 075017, 2011. 
[21] S.-C. Kwon, S.-H. Jeon, and H.-U. Oh, "Performance investigation of a novel pseudoelastic SMA mesh washer gear wheel with micro-jitter attenuation capability," Smart Materials and Structures, vol. 25, no. 5, Article ID 055004, 2016.

[22] H.-U. Oh, S.-H. Jeon, T.-H. Kim, and Y.-H. Kim, "Experimental feasibility study for micro-jitter attenuation of stepper-actuated $\mathrm{X}$-band antenna-pointing mechanism by using pseudoelastic SMA mesh washer," Smart Materials and Structures, vol. 24, no. 4, Article ID 045010, 2015.

[23] X. Yan and J. Nie, "Study of a new application form of shape memory alloy superelasticity," Smart Materials and Structures, vol. 12, no. 6, pp. N14-N23, 2003.

[24] G. Kang and D. Song, "Review on structural fatigue of NiTi shape memory alloys: pure mechanical and thermo-mechanical ones," Theoretical and Applied Mechanics Letters, vol. 5, no. 6, pp. 245-254, 2015.

[25] A. Isalgue, H. Soul, A. Yawny, and C. Auguet, "Functional fatigue recovery of superelastic cycled NiTi wires based on near $100^{\circ} \mathrm{C}$ aging treatments," in Proceedings of the 10th European Symposium on Martensitic Transformations (ESOMAT '15), vol. 33, September 2015.

[26] G. Park, D.-O. Lee, and J.-H. Han, "Development of multidegree-of-freedom microvibration emulator for efficient jitter test of spacecraft," Journal of Intelligent Material Systems and Structures, vol. 25, no. 9, pp. 1069-1081, 2014. 


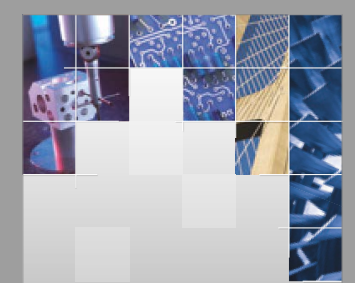

\section{Enfincering}
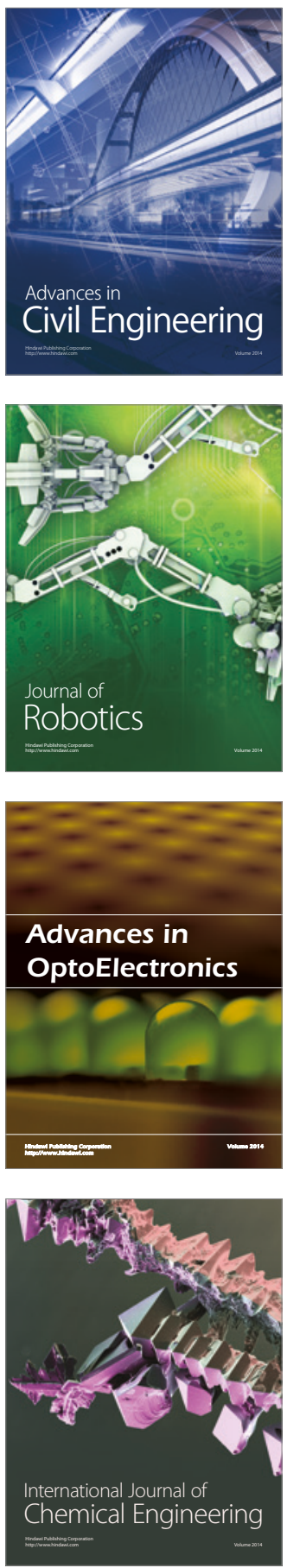

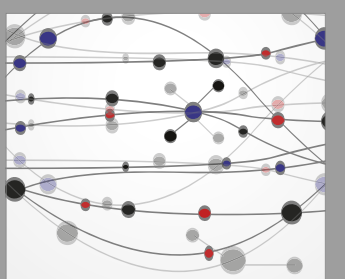

The Scientific World Journal

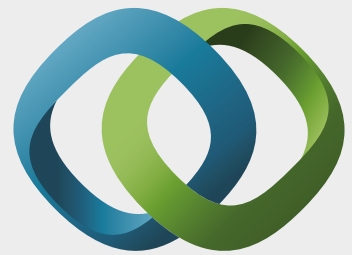

\section{Hindawi}

Submit your manuscripts at

https://www.hindawi.com
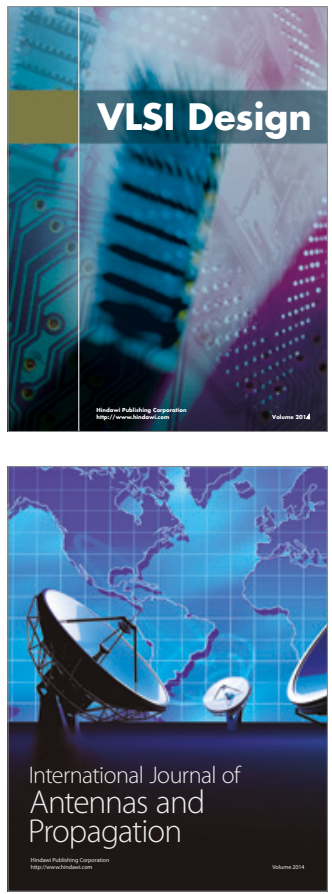

\section{Rotating}

Machinery
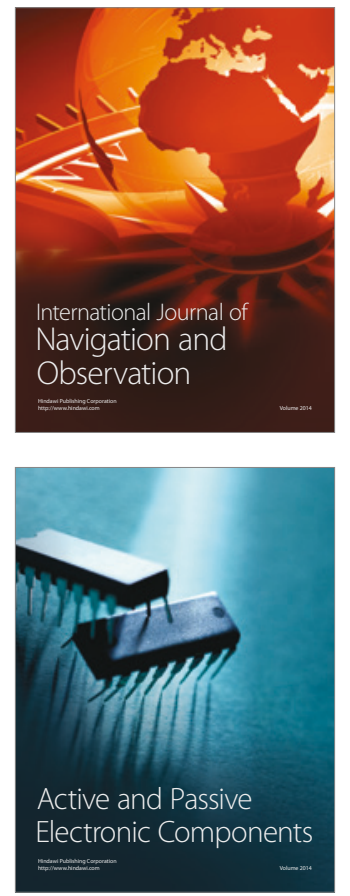
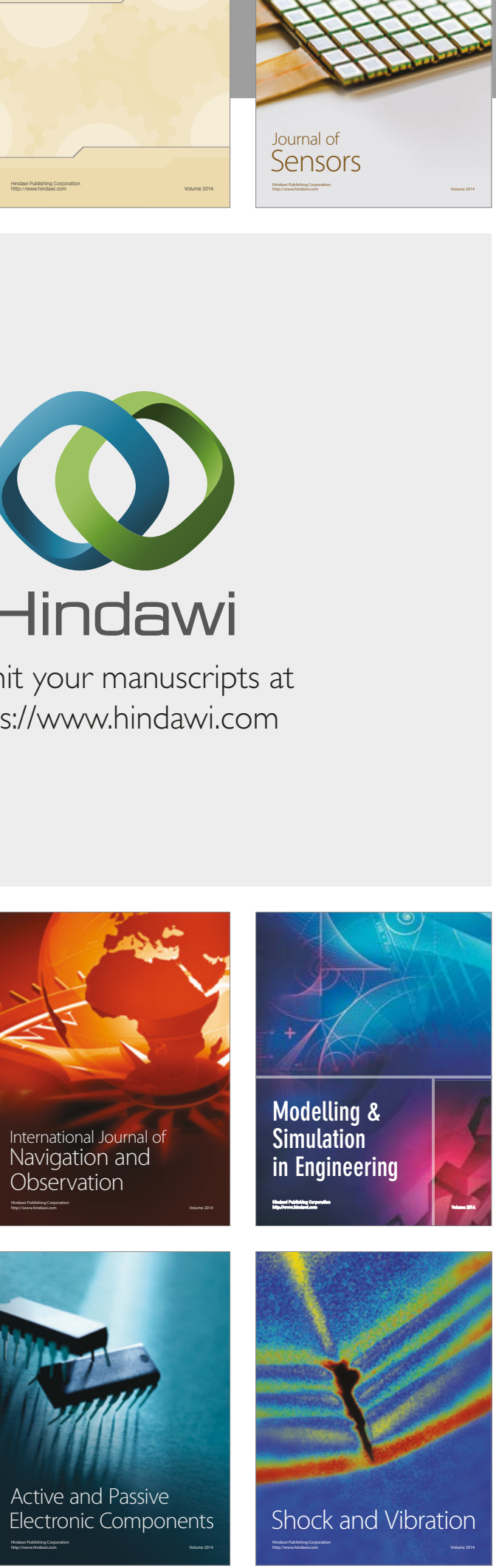
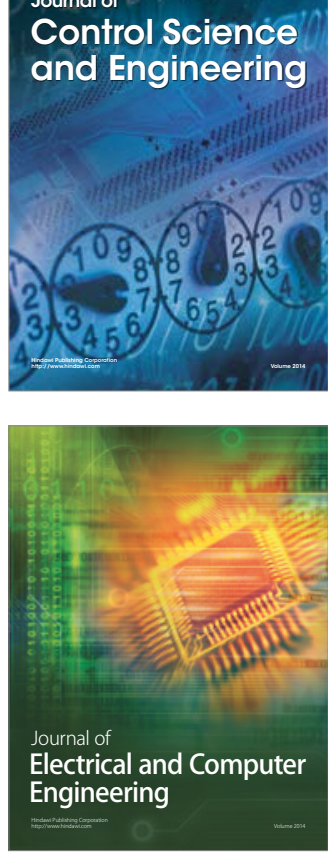

Distributed

Journal of

Control Science

and Engineering
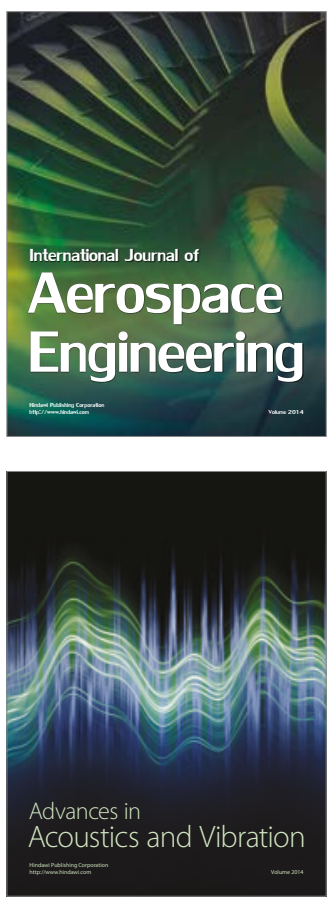

Sensor Networks 